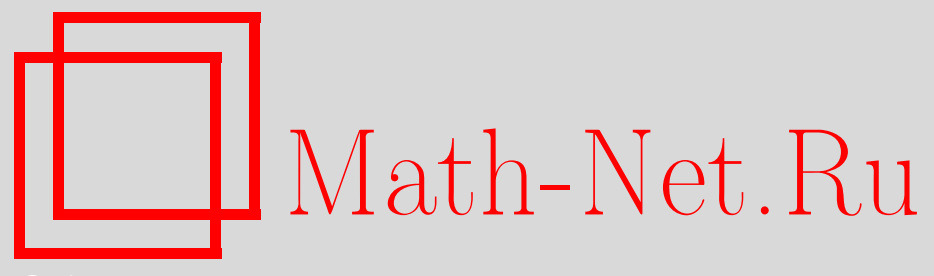

К. Фояш, М. С. Джолли, Р. Кравченко, Э. С. Тити, Единый подход к построению определяющих форм для двумерной системы уравнений Навье-Стокса: случай общих интерполирующих операторов, УМН, 2014, том 69, выпуск 2, 177-200

DOI: https://doi.org/10.4213/rm9583

Использование Общероссийского математического портала Math-Net.Ru подразумевает, что вы прочитали и согласны с пользовательским соглашением http://www.mathnet.ru/rus/agreement

Параметры загрузки:

IP : 54.162 .127 .20

26 апреля 2023 г., 15:33:43

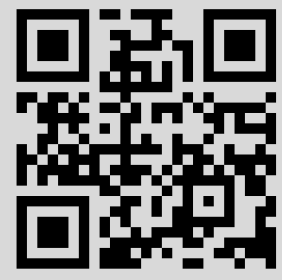




\title{
Единый подход к построению определяющих форм для двумерной системы уравнений Навье-Стокса: случай общих интерполирующих операторов
}

\author{
К. Фояш, М. С. Джолли, Р. Кравченко, Э. С. Тити
}

Мы показываем, что динамический поток при больших временах (глобальный аттрактор) двумерной системы Навье-Стокса вкладывается в динамический поток при больших временах обыкновенного дифференциального уравнения (называемого определяющей формой) в пространстве траекторий, изоморфном $C_{b}^{1}\left(\mathbb{R} ; \mathbb{R}^{N}\right)$ при достаточно большом $N$, где $N$ зависит от физических параметров системы Навье-Стокса. Предлагаемый единый подход основан на использовании интерполирующих операторов, построенных по произвольным определяющим параметрам системы уравнений Навье-Стокса (значениям в узлах, фурье-модам, конечным элементам объема, конечным элементам и т. п.). При таком едином подходе возникают два непосредственных небезынтересных следствия. Первое заключается в том, что определяющая форма имеет функцию Ляпунова, вследствие чего с неограниченным возрастанием времени ее решения сходятся к множеству стационарных решений определяющей формы. Вторым следствием является то, что эти стационарные решения определяющей формы можно однозначно отождествить с траекториями на глобальном аттракторе системы Навье-Стокса. Следует добавить, что данный подход является достаточно общим и применим практически без изменений к целому классу диссипативных динамических систем.

Библиография: 23 названия.

Ключевые слова: система уравнений Навье-Стокса, определяющие формы, определяющие моды, инерциальное многообразие, диссипативные динамические системы.

DOI: $10.4213 / \mathrm{rm} 9583$

\section{СОДЕРЖАНИЕ}

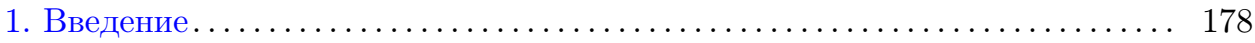

2. Функциональная постановка и система уравнений Навье-Стокса . . . . . 180

3. Определяющая форма и формулировка основных результатов ........ 185

3.1. Интерполирующие операторы...................... 185

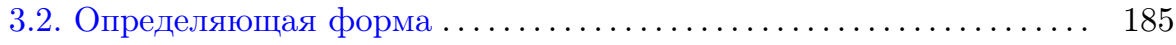

Работа выполнена при поддержке: первый автор - NSF (грант DMS-1109784), второй автор - NSF (гранты DMS-1008661, DMS-1109638), четвертый автор - NSF (гранты DMS-1009950, DMS-1109640, DMS-1109645) и Minerva Stiftung/Foundation.

(C) К. Фояш, М. С. Джолли, Р. Кравченко, Э. С. Тити, 2014 
4. Доказательство предложения $3.1 \ldots \ldots \ldots \ldots \ldots \ldots \ldots \ldots \ldots \ldots \ldots \ldots \ldots$

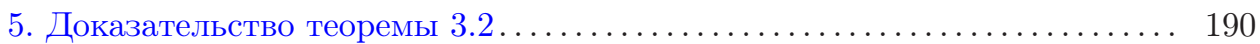

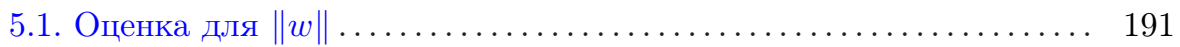

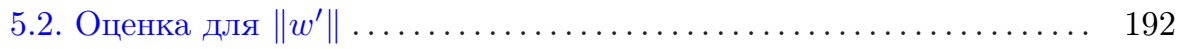

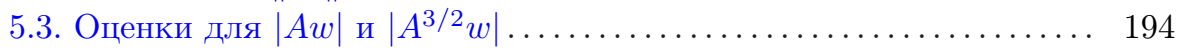

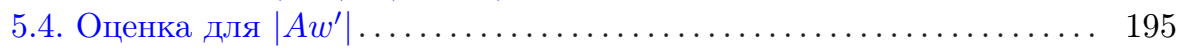

5.5. Липшицевость $w$ и $w^{\prime}$ в норме $D(A) \ldots \ldots \ldots \ldots \ldots \ldots \ldots \ldots \ldots . \ldots \ldots$

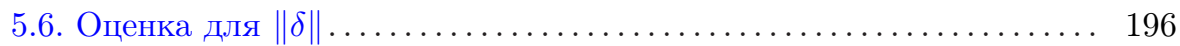

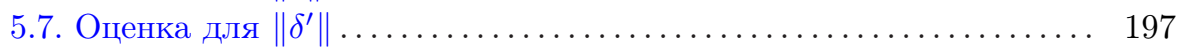

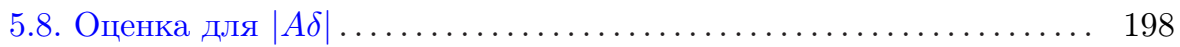

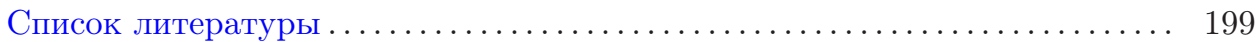

\section{1. Введение}

Двумерная система уравнений Навье-Стокса (2.1) и (2.2), являясь фундаментальной во многих моделях жидкости, вызывает также несомненный интерес по ряду причин чисто теоретического характера. В двумерной системе уравнений Навье-Стокса, которая обладает прямым каскадом энстрофии и обратным каскадом энергии, обнаруживаются более сложные явления турбулентности, чем в трехмерной системе Навье-Стокса [3], [19], [20]. Далее, в отличие от трехмерного уравнения, для двумерной системы Навье-Стокса теория существования глобальных решений является полной (см., например, [6], [22]). Фактически, динамический поток, соответствующий двумерной системе Навье-Стокса, при больших временах полностью содержится в глобалъном аттракторе $\mathscr{A}$ (см. (2.5)), являющемся компактным подмножеством бесконечномерного фазового пространства $H$ соленоидальных векторных полей с конечной энергией (см., например, [6], [10], [16], [22]). Точные оценки размерности глобального аттрактора в терминах соответствующих физических параметров были впервые получены в [7] (см. также [6], [21], [22] и приведенные там библиографические ссылки). Если бы существовало инерииальное многообразие $\mathscr{M}$ (т. е. липшицево конечномерное многообразие, инвариантное при движении вперед), притягивающее каждое ограниченное множество с экспоненциальной скоростью, то тогда имело бы место включение $\mathscr{A} \subset \mathscr{M}$ и в этом случае динамику на $\mathscr{A}$ можно было бы описать обыкновенным дифференциальным уравнением (называемым инерциальной формой) в конечномерном фазовом пространстве [6], [12], [13], [22]. Этого можно достичь сведением исходного эволюционного уравнения к уравнению, заданному на инерциальном многообразии $\mathscr{M}$. При этом вопрос о существовании инерциального многообразия для двумерной системы Навье-Стокса открыт с 80-х годов ХХ века!

Это достаточно удивительно, поскольку имеются даже еще более убедительные признаки конечномерного поведения двумерной системы Навье-Стокса. Решения из $\mathscr{A}$ определяются асимптотическим поведением достаточного (конечного) числа определяющих параметров. Если в пределе при $t \rightarrow \infty$ некоторое достаточно большое число низших фурье-мод (или значений в узлах, или конечных элементов объема) для двух решений из $\mathscr{A}$ сходятся друг к другу, 
то такие решения совпадают (см., например, [5] по поводу единой теории определяющих параметров и проекций). По крайней мере для случая фурье-мод это эквивалентно следующему:

если для двух полных траекторий на глобальном аттракторе их проекции $P_{m}$ на подпространство $m$ низших мод совпадают при достаточно большом $m$, то и сами траектории совпадают.

Введенное в [11] понятие определяющих мод (см. также [18] по поводу точных оценок числа определяющих мод) было использовано в [8] для построения системы обыкновенных дифференциальных уравнений в банаховом пространстве $X=C_{b}\left(\mathbb{R}, P_{m} H\right)$, описывающих эволюцию траекторий в $X$. Мы будем называть такую систему обыкновенных дифференциальных уравнений определяющей формой. Траектории на глобальном аттракторе $\mathscr{A}$ двумерной системы Навье-Стокса (2.2) отождествляются с решениями типа бегущей волны определяющей формы. По сути для определяющей формы разумно рассмотреть две временнь́е переменные: время эволюции обыкновенного дифференциального уравнения и исходную временну́ю переменную системы Навье-Стокса, с помощью которой мы теперь будем параметризовать полные траектории в $X$. Несмотря на то что определяющая форма обладает бесконечномерным фазовым пространством, векторное поле, определяющее эволюцию, является глобально липшицевым. Таким образом, определяющая форма является обыкновенным дифференциальным уравнением в истинном смысле. Ключевым моментом при построении определяющей формы в [8] являлось распространение определенного в (1.1) отображения $W: P_{m} \mathscr{S} \rightarrow\left(I-P_{m}\right) \mathscr{S}$ с множества $\mathscr{S}$ полных траекторий в $\mathscr{A}$ на все пространство $X$. При этом продолженное отображение оказывается липшицевым, через образы при отображении восстанавливаются высшие моды, а эволюционирующая траектория в $X$ представляет собой низшие моды.

Вводимая ниже определяющая форма обладает существенно иными свойствами. Это - система, обладающая функцией Ляпунова, причем ее стационарные решения в точности являются траекториями, лежащими на глобальном аттракторе двумерной системы Навье-Стокса. Она обладает достаточной общностью, что позволяет использовать ее с целым рядом определяющих параметров, включающих в себя значения в узлах и фурье-моды. Подход и стратегия с использованием определяющей формы достаточно универсальны и могут быть применены к другим диссипативным системам. Как и в случае определяющей формы в [8], ключевым моментом является продолжение отображения $W$, которое изначально определено только на проекциях траекторий из $\mathscr{A}$. Это достигается введением в систему Навье-Стокса члена обратной связи, что предлагалось в работах [1], [2]. При этом возникает интерполирующий оператор $J_{h}$, аппроксимирующий тождественное отображение на уровне $h$ (к примеру, $J_{h}$ может строиться по значениям в узлах, где $h$ - шаг сетки). Соответствующая конструкция раскрывается в разделе 3 , где также формулируются основные результаты. В разделе 2 даются предварительные вспомогательные сведения и приводятся неравенства, относящиеся к системе уравнений Навье-Стокса. В разделах 4 и 5 дается доказательство основных результатов. 


\section{2. Функциональная постановка и система уравнений Навье-Стокса}

Рассмотрим двумерную систему уравнений Навье-Стокса для несжимаемой жидкости

$$
\begin{gathered}
\frac{\partial u}{\partial t}-\nu \Delta u+(u \cdot \nabla) u+\nabla p=\Phi \\
\operatorname{div} u=0 \\
\int_{\Omega} u d x=0, \quad \int_{\Omega} \Phi d x=0 \\
u(0, x)=u_{0}(x)
\end{gathered}
$$

с периодическими граничными условиями и фундаментальной областью $\Omega=$ $[0, L]^{2}$. Поле скоростей $u$ и давление $p$ неизвестны, $\Phi$ - заданный возмущающий член, а $\nu>0$ - заданная постоянная вязкость.

Положим

$$
\begin{gathered}
\mathscr{V}=\left\{\phi: \phi-\mathbb{R}^{2}\right. \text {-значный тригонометрический полином, } \\
\left.\nabla \cdot \phi=0, \int_{\Omega} \phi(x) d x=0\right\} .
\end{gathered}
$$

Для любого подмножества $Z \subset L_{\text {per }}^{1}(\Omega)$ положим $\dot{Z}=\left\{\phi \in Z: \int_{\Omega} \phi(x) d x=0\right\}$. Далее, пусть $H$ и $V$ обозначают замыкание $\mathscr{V}$ в $\left(L_{\mathrm{per}}^{2}(\Omega)\right)^{2}$ и $\left(H_{\mathrm{per}}^{1}(\Omega)\right)^{2}$ соответственно. Скалярное произведение и норма на гильбертовых пространствах $\left(L_{\text {per }}^{2}(\Omega)\right)^{2}$ и $H$ обозначаются соответственно $(\cdot, \cdot)$ и $|\cdot|$; соответствующие скалярное произведение и норма на гильбертовых пространствах $\left(\dot{H}_{\mathrm{per}}^{1}(\Omega)\right)^{2}$ и $V$ обозначаются $((\cdot, \cdot))$ и $\|\cdot\|$. В частности, для $u, v \in\left(\dot{H}_{\mathrm{per}}^{1}(\Omega)\right)^{2}$

$$
((u, v))=\sum_{i, j=1}^{2} \int_{\Omega} \frac{\partial u_{i}(x)}{\partial x_{j}} \frac{\partial v_{i}(x)}{\partial x_{j}} d x .
$$

Через $V^{\prime}$ мы обозначим сопряженное пространство к $V$.

В приведенной выше функциональной постановке система Навье-Стокса может быть записана как эволюционное уравнение в гильбертовом пространстве $H$ (см. [6], [22])

$$
\begin{gathered}
\frac{d}{d t} u(t)+\nu A u(t)+B(u(t), u(t))=f, \quad t>0, \\
u(0)=u_{0} .
\end{gathered}
$$

Оператор Стокса $A$, билинейный оператор $B$ и сила $f$ задаются следующим образом:

$$
A=-\mathscr{P} \Delta, \quad B(u, v)=\mathscr{P}((u \cdot \nabla) v), \quad f=\mathscr{P} \Phi .
$$

Здесь $\mathscr{P}$ - ортогональный проектор Лере-Гельмгольца из $\left(\dot{L}_{\mathrm{per}}^{2}(\Omega)\right)^{2}$ на $H$, а $u$, $v$ - достаточно гладкие, так что значение оператора $B(u, v)$ имеет смысл. В данной работе мы будем предполагать, что $f \in V$.

Отметим, что $D(A)=\left(\dot{H}_{\text {per }}^{2}(\Omega)\right)^{2} \cap V$. Оператор $A$ является самосопряженным и имеет компактный обратный. Как следствие, в пространстве $H$ имеется 
ортонормальный базис $\left\{w_{j}\right\}_{j=1}^{\infty}$ из собственных функций оператора $A$. Именно, $A w_{j}=\lambda_{j} w_{j}$, где $0<\lambda_{1}=(2 \pi / L)^{2} \leqslant \lambda_{2} \leqslant \lambda_{3} \leqslant \cdots$ (см. [6], [22]). Степени $A^{\alpha}$ оператора $A$ имеют следующий вид:

$$
A^{\alpha} v=\sum_{j=1}^{\infty} \lambda_{j}^{\alpha}\left(v, w_{j}\right) w_{j}
$$

при этом все $A^{\alpha}$ коммутируют с $\mathscr{P}$. Также отметим, что $V:=D\left(A^{1 / 2}\right)$ и

$$
\|u\|=\left|A^{1 / 2} u\right|=\left(\sum_{j=1}^{\infty} \lambda_{j}\left(u, w_{j}\right)^{2}\right)^{1 / 2} .
$$

Хорошо известно, что система Навье-Стокса (2.2) имеет глобальный аттрактор

$$
\begin{gathered}
\mathscr{A}=\left\{u_{0} \in H: \text { существует решение } u\left(t, u_{0}\right) \text { системы }(2.2),\right. \\
\text { причем } \left.\sup _{t}\|u(t)\|<\infty \forall t \in \mathbb{R}\right\} ;
\end{gathered}
$$

т. е. $\mathscr{A}$ является максимальным ограниченным инвариантным подмножеством из $V$ в ходе динамической эволюции системы Навье-Стокса, или, эквивалентно, является минимальным компактным подмножеством из $V$, которое равномерно притягивает все ограниченные множества из $V$ в ходе динамической эволюции системы (2.2). В частности, известно, что

$$
\mathscr{A} \subset\left\{u \in V:\|u\| \leqslant G \nu \kappa_{0}\right\}, \quad \text { где } \quad G=\frac{|f|}{\nu^{2} \kappa_{0}^{2}} .
$$

Здесь $G$ - число Грасгофа (безразмерный физический параметр) и $\kappa_{0}=\lambda_{1}^{1 / 2}=$ $2 \pi / L$. По поводу указанных выше свойств см., например, [6], [10], [16], [22].

Ниже мы вводим ряд соотношений, которые выполняются для билинейного члена. В их число входят соотношения ортогональности

$$
\langle B(u, v), w\rangle=-\langle B(u, w), v\rangle, \quad u, v, w \in V
$$

(где $\langle\cdot, \cdot\rangle$ - соотношение двойственности между $V^{\prime}$ и $V$ ), и

$$
(B(u, u), A u)=0, \quad u \in D(A)
$$

(см., например, [6], [10], [22]). Из (2.7) вытекает (ср. [6], [22]), что

$$
(B(v, v), A u)+(B(v, u), A v)+(B(u, v), A v)=0, \quad u, v \in D(A) .
$$

Далее через $c, c_{A}, c_{B}, c_{L}, c_{S}, c_{T}, c_{1}, c_{2}, \tilde{c}_{1}, \tilde{c}_{2}, \ldots$ мы будем обозначать универсальные положительные безразмерные константы. Для того чтобы оценить нелинейный член, мы воспользуемся неравенством Агмона

$$
\|u\|_{\infty} \leqslant c_{\mathrm{A}}|u|^{1 / 2}|A u|^{1 / 2}, \quad u \in D(A),
$$

а также неравенствами Соболева и Ладыженской

$$
\begin{aligned}
\|u\|_{L^{4}(\Omega)} & \leqslant c_{\mathrm{S}}\|u\|_{H^{1 / 2}(\Omega)} \quad \text { для любого } u \in\left(\dot{H}_{\mathrm{per}}^{1 / 2}(\Omega)\right)^{2}, \\
\|u\|_{H^{1 / 2}(\Omega)} & \leqslant \tilde{c}_{\mathrm{L}}|u|^{1 / 2}\|u\|^{1 / 2} \quad \text { для любого } u \in\left(\dot{H}_{\mathrm{per}}^{1}(\Omega)\right)^{2},
\end{aligned}
$$


из которых следует, что

$$
\|u\|_{L^{4}(\Omega)} \leqslant c_{\mathrm{L}}|u|^{1 / 2}\|u\|^{1 / 2} \quad \text { для любого } u \in\left(\dot{H}_{\mathrm{per}}^{1}(\Omega)\right)^{2} .
$$

Нам также потребуются следующие варианты неравенства Пуанкаре:

$$
\kappa_{0}|v| \leqslant\|v\|, \quad u \in V, \quad \text { и } \quad \kappa_{0}\|v\| \leqslant|A v|, \quad u \in D(A),
$$

и неравенство Юнга

$$
a b \leqslant \frac{a^{p}}{p}+\frac{b^{q}}{q} \quad \text { при } \quad a, b, p, q>0 \quad \text { и } \quad \frac{1}{p}+\frac{1}{q}=1 .
$$

Применяя (2.13), мы имеем

$$
|(B(u, v), w)| \leqslant c_{\mathrm{S}}|u|^{1 / 2}\|u\|^{1 / 2}\|v\|^{1 / 2}|A v|^{1 / 2}|w| \quad \forall u \in V, v \in D(A), w \in H,
$$

и далее, по (2.10),

$$
|(B(u, v), w)| \leqslant c_{\mathrm{A}}|u|^{1 / 2}|A u|^{1 / 2}\|v\||w| \quad \forall u \in D(A), v \in V, w \in H .
$$

При этом (см. [23])

$$
|(B(w, u), v)| \leqslant c_{T}\|w\|\|u\|\left(\log \frac{e|A u|}{\kappa_{0}\|u\|}\right)^{1 / 2}|v| \quad \forall u \in D(A), v \in H, w \in V .
$$

Используя неравенство Брезиса-Галлуэ [4], мы получаем

$$
|(B(w, u), v)| \leqslant c_{B}\|w\|\|u\|\left(\log \frac{e|A w|}{\kappa_{0}\|w\|}\right)^{1 / 2}|v| \quad \forall u \in V, v \in H, w \in D(A)
$$

(по поводу другого доказательства см. [23]).

Также мы будем использовать следующее модифицированное неравенство Гронуолла ([17]; см. также [10]).

Лемма 2.1. Пусть $\alpha, \beta$ - локально интегрируемъе действительные функчии на $(0, \infty)$, для которых при некотором $T \in(0, \infty)$

$$
\liminf _{t \rightarrow \infty} \frac{1}{T} \int_{t}^{t+T} \alpha(\tau) d \tau=\gamma>0, \quad \limsup _{t \rightarrow \infty} \frac{1}{T} \int_{t}^{t+T} \alpha^{-}(\tau) d \tau<\infty
$$

$u$

$$
\lim _{t \rightarrow \infty} \frac{1}{T} \int_{t}^{t+T} \beta^{+}(\tau) d \tau=0
$$

где $\alpha^{-}=\max \{-\alpha, 0\}$ и $\beta^{+}=\max \{\beta, 0\}$. Пусть также $\xi-$ абсолютно непрерывная неотрицательная функция на $(0, \infty)$, для которой

$$
\frac{d}{d t} \xi+\alpha \xi \leqslant \beta \quad \text { n. в. на }(0, \infty) .
$$

Тогда $\xi(t) \rightarrow 0$ при $t \rightarrow \infty$.

Ниже лемма 2.1 будет применяться в комбинации со следующими оценками усредненных решений (см. [17], [18]). 
ПреДЛОЖЕНИЕ 2.2. Пусть $и$ - решение системы Навъе-Стокса (2.2) и пусть $T=\left(\nu \kappa_{0}^{2}\right)^{-1}$. Тогда

$$
\limsup _{t \rightarrow \infty} \frac{1}{T} \int_{t}^{t+T}|A u(\tau)|^{2} d \tau \leqslant 2 \nu^{2} \kappa_{0}^{4} G^{2} .
$$

Если $u \in \mathscr{A}$, mо

$$
\limsup _{t_{0} \rightarrow-\infty} \frac{1}{t-t_{0}} \int_{t_{0}}^{t}|A u(\tau)|^{2} d \tau \leqslant \nu^{2} \kappa_{0}^{4} G^{2} \quad \text { nри всех } t \in \mathbb{R} .
$$

Более того, из неравенства Коши-Буняковского вытекает, что

$$
\limsup _{t_{0} \rightarrow-\infty} \frac{1}{t-t_{0}} \int_{t_{0}}^{t}|A u(\tau)| d \tau \leqslant \nu \kappa_{0}^{2} G \quad \text { при всех } t \in \mathbb{R} .
$$

ПреДЛОЖеНИЕ 2.3. Пусть $и(t)$ - решение системъ Навъе-Стокса (2.2). Тогда

$$
\limsup _{t \rightarrow \infty}\|u(t)\| \leqslant \nu \kappa_{0} G \quad u \quad \limsup _{t \rightarrow \infty}|A u(t)| \leqslant c \nu \kappa_{0}^{2} G^{3} .
$$

В частности,

$$
\|u(t)\| \leqslant \nu \kappa_{0} G \quad u \quad|A u| \leqslant c_{0} \nu \kappa_{0}^{2} G^{3} \quad \forall u \in \mathscr{A} .
$$

Более того, решения из глобального аттрактора $\mathscr{A}$ являются аналитическими по переменной времени в полосе шириной $\delta_{\text {Time }} \geqslant c /\left(\nu \kappa_{0}^{2} G^{4}\right)$, симметричной относительно действительной прямой. При этом по формуле Коши из данных оценок следует, что

$$
\sup _{t \in \mathbb{R}}\left\|\frac{d u}{d t}(t)\right\| \leqslant c \nu^{2} \kappa_{0}^{3} G^{5} \quad u \quad \sup _{t \in \mathbb{R}}\left|A \frac{d u}{d t}(t)\right| \leqslant c \nu^{2} \kappa_{0}^{4} G^{7} .
$$

Идейно доказательство предложения 2.3 восходит к [6]. Однако новые точные оценки из предложения 2.3 получены в [9].

Нашей дальнейшей целью является получение следующих двух оценок для $A^{1 / 2} B(u, v)$.

ПреДЛОЖЕНИЕ 2.4. Пусть $u \in D\left(A^{3 / 4}\right), v \in D(A)$. Тогда

$$
\left|A^{1 / 2} B(u, v)\right| \leqslant c\left(\left|A^{3 / 4} u\right|\left|A^{3 / 4} v\right|+|u|^{1 / 2}|A u|^{1 / 2}|A v|\right) .
$$

Далее, пусть $u \in V, v \in D\left(A^{3 / 2}\right)$. Тогда

$$
\left|A^{1 / 2} B(u, v)\right| \leqslant c\left(\left|A^{1 / 2} u\right|\left|A^{1 / 2} v\right|^{1 / 2}\left|A^{3 / 2} v\right|^{1 / 2}+\left|A^{1 / 4} u\right|\left|A^{5 / 4} v\right|\right) .
$$

ДокАЗАТЕЛьство. Для начала отметим, что

$$
\left|A^{1 / 2} \mathscr{P} \phi\right| \leqslant|\nabla \phi| \quad \text { для любой } \phi \in\left(\dot{H}_{\mathrm{per}}^{1}(\Omega)\right)^{2} .
$$

Из неравенства Соболева (2.11) имеем

$$
\left(\left(\partial_{i} u \cdot \nabla\right) v, w\right) \leqslant\left\|\partial_{i} u\right\|_{L^{4}}\|\nabla v\|_{L^{4}}|w| \leqslant\left\|\partial_{i} u\right\|_{H^{1 / 2}}\|\nabla v\|_{H^{1 / 2}}|w| .
$$


Далее, из $(2.26),(2.27),(2.10)$ и (2.12) вытекает, что

$$
\begin{aligned}
\left|A^{1 / 2} B(u, v)\right| & \leqslant \sum_{i=1,2}\left|\partial_{i}(u \cdot \nabla) v\right|=\sum_{i=1,2}\left|\left(\partial_{i} u \cdot \nabla\right) v\right|+\sum_{i=1,2}\left|(u \cdot \nabla) \partial_{i} v\right| \\
& \leqslant c \sum_{i=1,2}\left(\left\|\partial_{i} u\right\|_{H^{1 / 2}}\|\nabla v\|_{H^{1 / 2}}+\|u\|_{L^{\infty}}\left|\nabla \partial_{i} v\right|\right) \\
& \leqslant c\left(\left|A^{3 / 4} u\right|\left|A^{3 / 4} v\right|+|u|^{1 / 2}|A u|^{1 / 2}|A v|\right)
\end{aligned}
$$

И

$$
\begin{aligned}
\left|A^{1 / 2} B(u, v)\right| & \leqslant \sum_{i=1,2}\left|\partial_{i}(u \cdot \nabla) v\right|=\sum_{i=1,2}\left|\left(\partial_{i} u \cdot \nabla\right) v\right|+\sum_{i=1,2}\left|(u \cdot \nabla) \partial_{i} v\right| \\
& \leqslant c \sum_{i=1,2}\left(\left|\partial_{i} u\right|\|\nabla v\|_{L^{\infty}}+\|u\|_{H^{1 / 2}}\left\|\nabla \partial_{i} v\right\|_{H^{1 / 2}}\right) \\
& \leqslant c\left(\left|A^{1 / 2} u\right|\left|A^{1 / 2} v\right|^{1 / 2}\left|A^{3 / 2} v\right|^{1 / 2}+\left|A^{1 / 4} u\right|\left|A^{5 / 4} v\right|\right) .
\end{aligned}
$$

Отталкиваясь от доказательства неравенства Брезиса-Галлуэ [4], мы ниже устанавливаем оценку для $L^{\infty}$-нормы, которая в свою очередь нам понадобится для оптимизации оценок.

Лемма 2.5. Пусть $\phi \in \dot{H}_{\text {per }}^{2}(\Omega)$. Тогда для любого $N \in \mathbb{R}^{+}$

$$
\|\phi\|_{L^{\infty}} \leqslant \mathscr{L}_{N}|\nabla \phi|+\left(\sqrt{\pi} \kappa_{0} N\right)^{-1}|\Delta \phi|,
$$

где $\mathscr{L}_{N}=(8+2 \pi \log N)^{1 / 2}(2 \pi)^{-1}$.

ДокАЗАтЕльство. Для начала отметим, что

$$
\begin{aligned}
\sum_{1 \leqslant|k|^{2} \leqslant N^{2}} \frac{1}{|k|^{2}} & =6+\sum_{3 \leqslant|k|^{2} \leqslant N^{2}} \frac{1}{|k|^{2}} \\
& \leqslant 6+4 \int_{2}^{N} \frac{d x}{x^{2}}+\int_{1}^{N} \int_{0}^{2 \pi} \frac{1}{r^{2}} r d \theta d r \leqslant 8+2 \pi \log N
\end{aligned}
$$

и далее, при $N \geqslant 3$,

$$
\sum_{N+1 \leqslant|k|} \frac{1}{|k|^{4}} \leqslant 4 \frac{1}{(N+1)^{4}}+4 \int_{N+1}^{\infty} \frac{d x}{x^{4}}+\int_{N-1}^{\infty} \int_{0}^{2 \pi} \frac{1}{r^{4}} r d \theta d r \leqslant \frac{4 \pi}{N^{2}} .
$$

Используя неравенство Коши-Буняковского и равенство Парсеваля, окончательно имеем

$$
\begin{aligned}
\|\phi\|_{L^{\infty}} \leqslant & \sum_{1 \leqslant|k| \leqslant N}\left|\hat{\phi}_{k}\right|+\sum_{|k| \geqslant N+1}\left|\hat{\phi}_{k}\right| \\
\leqslant & {\left[\sum_{1 \leqslant|k| \leqslant N} \frac{1}{|k|^{2}}\right]^{1 / 2}\left[\sum_{1 \leqslant|k| \leqslant N}|k|^{2}\left|\hat{\phi}_{k}\right|^{2}\right]^{1 / 2} } \\
& +\left[\sum_{|k| \geqslant N+1} \frac{1}{|k|^{4}}\right]^{1 / 2}\left[\sum_{|k| \geqslant N+1}|k|^{4}\left|\hat{\phi}_{k}\right|^{2}\right]^{1 / 2} \\
\leqslant & (8+2 \pi \log N)^{1 / 2} \frac{|\nabla \phi|}{2 \pi}+\frac{2 \sqrt{\pi}}{N} \frac{|\Delta \phi|}{2 \pi \kappa_{0}} .
\end{aligned}
$$




\section{3. Определяющая форма и формулировка основных результатов}

3.1. Интерполирующие операторы. В данном разделе мы предлагаем унифицированный подход к использованию определяющих параметров (моды, узлы, элементы объема и т. д.) при помощи их представления через интерполирующие операторы, которые аппроксимируют тождественное отображение.

Пусть $J=J_{h}:\left(\dot{H}_{\text {per }}^{2}(\Omega)\right)^{2} \rightarrow\left(\dot{C}_{\text {per }}^{\infty}(\Omega)\right)^{2}$ - оператор конечного ранга, аппроксимирующий тождественное отображение в следующем смысле: для каждой $\phi \in\left(\dot{H}_{\text {per }}^{2}(\Omega)\right)^{2}$ имеет место включение $J \phi \in\left(C_{\text {per }}^{\infty}(\Omega)\right)^{2}$ и $J \phi$ имеет нулевое пространственное среднее. Дополнительно мы предположим, что

$$
\begin{gathered}
|J \phi-\phi| \leqslant c_{1} h|\nabla \phi|+c_{2} h^{2}|\Delta \phi|, \\
|\nabla(J \phi-\phi)| \leqslant \tilde{c}_{1}|\nabla \phi|+\tilde{c}_{2} h|\Delta \phi| .
\end{gathered}
$$

Здесь $h$ - малый параметр, определяющий порядок аппроксимации. Ранг оператора $J_{h}$ имеет порядок $L / h \geqslant 1$. K примеру, такие интерполяционные многочлены индуцируются определяющими параметрами системы Навье-Стокса, такими как определяющие моды, узлы, элементы объема, проекции конечных элементов и т. д. (см., например, [5], [11], [14], [15], [17], [18] и приведенные в этих работах ссылки). Наиболее простым примером таких интерполирующих операторов является оператор проекции $J_{h}=P_{m}$ на подпространство $\operatorname{span}\left\{w_{1}, w_{2}, \ldots, w_{m}\right\}$, где $h=\lambda_{m+1}^{-1}$. В приложении к работе [1] приводятся явные примеры интерполирующих операторов, основанных на значениях в узлах и удовлетворяющих условиям (3.1), (3.2). Также стоит отметить, что в [5] предлагается общий подход к исследованию динамики при больших временах системы Навье-Стокса с использованием интерполирующих многочленов, удовлетворяющих (3.1) .

3.2. Определяющая форма. В данном пункте мы вводим определяющую форму, индуцированную интерполирующими операторами $J_{h}$. В следующем утверждении раскрывается связь между динамикой при больших временах системы Навье-Стокса (2.2) и определяющей формы.

ПреДЛОЖЕНИЕ 3.1. Пусть $G \geqslant 1$, и пусть $u(s), s \in \mathbb{R},-$ решение системъ Навъе-Стокса (2.2), лежащее в глобальном аттракторе $\mathscr{A}$. Предположим, что $w \in C_{b}(\mathbb{R} ; V) \cap L_{\text {loc }}^{2}(\mathbb{R} ; D(A))$ такова, что $d w / d s \in L_{\text {loc }}^{2}(\mathbb{R} ; H)$ u

$$
\frac{d w}{d s}+\nu A w+B(w, w)=f-\mu \nu \kappa_{0}^{2} \mathscr{P} J(w-u) .
$$

Тогда $w=u$, если

$$
\mu>6 c_{T} G \log \left(c_{3} G\right), \quad \text { əде } \quad c_{3}=\left(2 c_{T} c_{0}\right)^{1 / 3},
$$

a $h$ достаточно мало и удовлетворяет неравенству

$$
2 \mu \kappa_{0}^{2} c_{J} h^{2} \leqslant 1, \quad \text { əде } \quad c_{J}=c_{1}+\frac{c_{2}^{2}}{2}
$$

$c_{T}, c_{0}, c_{1}, c_{2}$ - константы из (2.18), (2.23), (3.1) соответственно. 
Доказательство предложения 3.1 дается в разделе 4. Отметим, что существование решений уравнения (3.3) в предложении 3.1 обеспечивается теоремой 3.2 .

Далее мы вводим фазовое пространство для динамики, описываемой определяющей формой. Рассмотрим пространство

$$
X=C_{b}^{1}\left(\mathbb{R}, J\left(\dot{H}_{\mathrm{per}}^{2}(\Omega)\right)^{2}\right),
$$

на котором определены две нормы

$$
\begin{aligned}
\|v\|_{X} & =\frac{\sup _{s \in \mathbb{R}}\|v(s)\|}{\nu \kappa_{0}}+\frac{\sup _{s \in \mathbb{R}}\left\|v^{\prime}(s)\right\|}{\nu^{2} \kappa_{0}^{3}}, \\
\|v\|_{X, 0} & =\frac{\sup _{s \in \mathbb{R}}\|v(s)\|}{\nu \kappa_{0}} .
\end{aligned}
$$

Пусть $v$ - заданный элемент пространства $X$. Рассмотрим уравнение

$$
\frac{d w}{d s}+\nu A w+B(w, w)=f-\mu \nu \kappa_{0}^{2} \mathscr{P}(J w-v) .
$$

В теореме 3.2 мы показываем, что при определенных условиях на параметры $\mu$ и $h$ (которые зависят от нормы $\|v\|_{X}$ ) уравнение (3.6) имеет единственное ограниченное решение $w(s)$, глобальное по $s \in \mathbb{R}$.

Tеорема 3.2. Пусть $f \in V$. Рассмотрим шар $\mathscr{B}_{X}^{\rho}(0)=\left\{v \in X:\|v\|_{X} \leqslant \rho\right\}$ при некотором $\rho>0$. Пусть числа $K$ и н таковы, что

$$
K \geqslant\left(2 \rho^{2}+\frac{G^{2}}{\mu}+1\right)^{1 / 2}
$$

$u$

$$
c_{4} K^{2} \log \left(c_{5} K^{2}\right)<\mu<2 c_{4} K^{2} \log \left(c_{5} K^{2}\right),
$$

где $c_{4}=80\left(c_{T}+c_{B}+1\right)^{2}, c_{5}=\sqrt{8}\left(c_{T}+c_{B}+1\right)$. Выберем достаточно малое $h$, для которого

$$
2 \mu h^{2} \kappa_{0}^{2}\left(c_{1}^{2}+c_{2}\right)<\frac{1}{2}
$$

Тогда для любого $v \in \mathscr{B}_{X}^{\rho}(0)$ уравнение (3.6) имеет единственное решение $w(s)$, существующее глобально по $s \in \mathbb{R}$ и обладающее следующими свойствами:

(i) $\sup _{s \in \mathbb{R}}\|w(s)\| \leqslant \nu^{2} \kappa_{0}^{2} K^{2}$,

(iii) $\sup _{s \in \mathbb{R}}|A w(s)| \leqslant \nu \kappa_{0}^{2} C(K)$,

(v) $\sup _{s \in \mathbb{R}}\left|A w^{\prime}(s)\right| \leqslant \kappa_{0} C(K)\left(\|f\|+\nu^{2} \kappa_{0}^{3}\right)$. (ii) $\sup _{s \in \mathbb{R}}\left\|w^{\prime}(s)\right\| \leqslant \nu^{2} \kappa_{0}^{3} C(K)$

(iv) $\sup _{s \in \mathbb{R}}\left|A^{3 / 2} w(s)\right| \leqslant \frac{\|f\|}{\nu}+\nu \kappa_{0}^{3} C(K)$,

Далее, пусть $v_{1}, v_{2} \in \mathscr{B}_{X}^{\rho}(0)$ и $w_{1}, w_{2}$ - соответствующие решения уравнения (3.6). Положим $\gamma=v_{1}-v_{2} u \delta=w_{1}-w_{2}$. Тогда:

$$
\begin{array}{ll}
\text { (vi) } \sup _{s \in \mathbb{R}}\|\delta(s)\| \leqslant 4 \nu \kappa_{0}\|\gamma\|_{X}, \quad \text { (vii) } \sup _{s \in \mathbb{R}}\left\|\delta^{\prime}(s)\right\| \leqslant \nu^{2} \kappa_{0}^{3} C(K)\|\gamma\|_{X}, \\
\text { (viii) } \sup _{s \in \mathbb{R}}|A \delta(s)| \leqslant \nu \kappa_{0}^{2} C(K)\|\gamma\|_{X},
\end{array}
$$

где $C(K)=c \exp \left(c K^{2} \log K\right)$ с некоторой универсальной постоянной $c>0$. 
Доказательство теоремы 3.2 будет дано в разделе 5 .

Следующий результат непосредственно вытекает из теоремы 3.2.

СЛЕДСтвиЕ 3.3. В условиях теоремъ 3.2 существует непрерывное по Липшицу отображение

$$
W: \mathscr{B}_{X}^{\rho}(0) \rightarrow C_{b}^{1}(\mathbb{R} ; D(A))
$$

со следующими свойствами:

(i) $W(v)(s)=w(s)$ при каждом $v \in \mathscr{B}_{X}^{\rho}(0)$ и всех $s \in \mathbb{R}$, где $w(s)-$ единственное решение уравнения (3.6), соответствующее заданному $v(s)$;

(ii) при каждых $v_{1}, v_{2} \in \mathscr{B}_{X}^{\rho}(0)$

$$
\begin{aligned}
& \frac{1}{\kappa_{0}} \sup _{s \in \mathbb{R}}\left|A\left(W\left(v_{1}\right)(s)-W\left(v_{2}\right)(s)\right)\right|+\sup _{s \in \mathbb{R}}\left\|W\left(v_{1}\right)(s)-W\left(v_{2}\right)(s)\right\| \\
& \quad+\frac{1}{\nu \kappa_{0}^{2}} \sup _{s \in \mathbb{R}}\left\|\frac{d}{d s}\left(W\left(v_{1}\right)(s)-W\left(v_{2}\right)(s)\right)\right\| \leqslant \nu \kappa_{0} C(K)\left\|v_{1}-v_{2}\right\|_{X} .
\end{aligned}
$$

Отображение $W(v)$ из следствия 3.3 играет важную роль при определении определяющей формы. Более точно, пусть $u^{*}-$ стационарное решение системы Навье-Стокса (2.2). Наша определяющая форма - это уравнение

$$
\frac{d v}{d t}=F(v)=-\|v-J W(v)\|_{X, 0}^{2}\left(v-J u^{*}\right) .
$$

Некоторые свойства уравнения (3.10) будут раскрыты ниже в теореме 3.5, но сначала нам потребуется следующий результат.

ПреДЛОЖЕНИЕ 3.4. Пустъ $G \geqslant 1$. Тогда для любого $u \in \mathscr{A}$

$$
\|J(u)\| \leqslant c^{*} \nu \kappa_{0} G^{3} \quad u \quad\left\|J\left(u^{\prime}\right)\right\| \leqslant c^{*} \nu^{2} \kappa_{0}^{3} G^{7} .
$$

Как следствие,

$$
\|J(u)\|_{X} \leqslant c^{*} G^{7}=: R .
$$

ДокАЗАТЕЛьство. Так как $\|J(\phi)\|=|\nabla J(\phi)|$, то мы можем применить (3.2) и воспользоваться тем фактом, что $h \kappa_{0} \leqslant 2 \pi$ в силу $(3.9)$, что вместе с предложением 2.3 завершает доказательство.

Теорема 3.5. Пусть $G \geqslant 1$. Предположим, что выполнены условия теоремы 3.2 с $\rho=4 R$, где $R:=c^{*} G^{7}$ определено в предложении 3.4. Тогда справедливы следующие утверждения.

(i) Векторное поле в определяющей форме (3.10) является липшицевым отображением из шара $\mathscr{B}_{X}^{\rho}(0)=\left\{v \in X:\|v\|_{X}<\rho\right\}$ в пространство $X$. Как следствие, (3.10) является обыкновенным дифференциальным уравнением в пространстве $X$ и для него имеет место существование и единственность решения на коротком интервале времени для начальных данных из $\mathscr{B}_{X}^{\rho}(0)$.

(ii) ШШар $\mathscr{B}_{X}^{3 R}\left(J\left(u^{*}\right)\right)=\left\{v \in X:\left\|v-J\left(u^{*}\right)\right\|_{X}<3 R\right\} \subset \mathscr{B}_{X}^{\rho}(0)$ инвариантен при возрастании времени в ходе динамической эволюиии определяющей формы (3.10). Как следствие, для (3.10) имеет место существование и единственность решения на коротком интервале времени для всех начальных данных из $\mathscr{B}_{X}^{3 R}\left(J\left(u^{*}\right)\right)$. 
(iii) Каждое решение (3.10) с начальными данными из $\mathscr{B}_{X}^{3 R}\left(J\left(u^{*}\right)\right)$ сходится при $t \rightarrow \infty$ к множеству стационарных решений уравнения (3.10).

(iv) Все содержашиеся в шаре $\mathscr{B}_{X}^{\rho}(0)$ стационарные решения определяющей формы (3.10) имеют вид $v(s)=J u(s)$ при всех $s \in \mathbb{R}$, где $u(s)-$ траектория, лежащая на глобальном аттракторе $\mathscr{A}$ системы Навъе-Стокса (2.2).

ДокАзАтельство. Чтобы доказать существование решения на коротком интервале времени, достаточно показать, что векторное поле (3.10) липшицево. Положим $F(v)=-g^{2}(v)\left(v-u^{*}\right)$, где $g(v)=\|v-J W(v)\|_{X, 0}$. Поскольку

$$
\left\|F\left(v_{1}\right)-F\left(v_{2}\right)\right\|_{X} \leqslant\left|g^{2}\left(v_{1}\right)-g^{2}\left(v_{2}\right)\right|\left\|v_{1}-u^{*}\right\|_{X}+\left|g^{2}\left(v_{2}\right)\right|\left\|v_{1}-v_{2}\right\|_{X},
$$

то нам достаточно показать, что отображение $g: \mathscr{B}_{X}^{\rho}(0) \rightarrow \mathbb{R}$ липшицево. Отметим, что

$$
\begin{array}{r}
\left|\left\|v_{1}-J W\left(v_{1}\right)\right\|_{X, 0}-\left\|v_{2}-J W\left(v_{2}\right)\right\|_{X, 0}\right| \leqslant\left\|v_{1}-J W\left(v_{1}\right)-\left[v_{2}-J W\left(v_{2}\right)\right]\right\|_{X, 0} \\
\leqslant\left\|v_{1}-v_{2}\right\|_{X, 0}+\left\|J W\left(v_{1}\right)-J W\left(v_{2}\right)\right\|_{X, 0}
\end{array}
$$

при $v_{1}, v_{2} \in \mathscr{B}_{X}^{\rho}(0)$. Далее, из (3.2) и неравенства треугольника вытекает, что

$$
\begin{aligned}
\left\|J W\left(v_{1}\right)-J W\left(v_{2}\right)\right\|_{X, 0} \leqslant\left(\tilde{c}_{1}+1\right) \sup _{s \in \mathbb{R}} & \left\|W\left(v_{1}\right)(s)-W\left(v_{2}\right)(s)\right\| \\
& +\tilde{c}_{2} h \sup _{s \in \mathbb{R}}\left|A\left(W\left(v_{1}\right)(s)-W\left(v_{2}\right)(s)\right)\right| .
\end{aligned}
$$

Теперь из следствия 3.3 и неравенства $h \kappa_{0} \leqslant 2 \pi$ имеем

$$
\left|\left\|v_{1}-J W\left(v_{1}\right)\right\|_{X, 0}-\left\|v_{2}-J W\left(v_{2}\right)\right\|_{X, 0}\right| \leqslant C(K)\left\|v_{1}-v_{2}\right\|_{X},
$$

где $K$ удовлетворяет неравенству (3.7) с $\rho=4 R$. Итак, (i) доказано.

Из предложения 3.4 вытекает, что $\mathscr{B}_{X}^{3 R}\left(J\left(u^{*}\right)\right) \subset \mathscr{B}_{X}^{\rho}(0)$. Таким образом, нами доказано существование решения (3.10) на коротком интервале времени для начальных данных из $\mathscr{B}_{X}^{3 R}\left(J\left(u^{*}\right)\right)$. Теперь (ii) вытекает из диссипативности (3.10). Именно, для каждого фиксированного $s \in \mathbb{R}$ мы имеем

$$
\begin{aligned}
\frac{d\left\|v(t ; s)-J\left(u^{*}\right)\right\|^{2}}{d t} & =-2\|v-J W(v)\|_{X, 0}^{2}\left\|v(t ; s)-J\left(u^{*}\right)\right\|^{2}, \\
\frac{d\left\|v^{\prime}(t ; s)\right\|^{2}}{d t} & =-2\|v-J W(v)\|_{X, 0}^{2}\left\|v^{\prime}(t ; s)\right\|^{2},
\end{aligned}
$$

где штрих обозначает дифференцирование по $s$. Отсюда вытекает, что шар $\mathscr{B}_{X}^{3 R}\left(J\left(u^{*}\right)\right)$ инвариантен при движении вперед при всех $t \geqslant 0$, что одновременно доказывает (ii) и (iii) (см. приводимое ниже обоснование стационарных решений для определяющей формы (3.10)).

Для доказательства утверждения (iv) отметим, что стационарные решения уравнения (3.10) в шаре $\mathscr{B}_{X}^{\rho}(0)$ имеют вид $v=J u^{*}$ или $v \in \mathscr{B}_{X}^{\rho}(0)$, причем $\|v-J W(v)\|_{X, 0}=0$. $\mathrm{B}$ первом случае $u^{*} \in \mathscr{A}$, поскольку $u^{*}$ является стационарным решением системы Навье-Стокса (2.2). Во втором случае $v(s)=J W(v)(s)$, т. е. $v(s)=J(w(s))$ при всех $s \in \mathbb{R}$, где $w(s)$ - решение уравнения (3.6). В этом случае из (3.6) вытекает, что $w(s)$ - ограниченное решение системы Навье-Стокса (2.2). Теперь из (2.6) следует, что $w(\cdot)$ - траектория на глобальном аттракторе $\mathscr{A}$ системы Навье-Стокса. Обратно, так как $\rho=4 R$, 
то из предложения 3.4 имеем, что $J(\mathscr{A}) \subset \mathscr{B}_{X}^{3 R}\left(J\left(u^{*}\right)\right) \subset \mathscr{B}_{X}^{\rho}(0)$. Таким образом, для каждой траектории $u(\cdot) \subset \mathscr{A}$ из предложения 3.1 и уравнения (3.6) следует, что $u(s)=W(J u)(s)$ при всех $s \in \mathbb{R}$. В частности, $J u=J W(J u)$ и, значит, $J u$ - стационарное решение $(3.10)$ в шаре $\mathscr{B}_{X}^{\rho}(0)$.

\section{4. Доказательство предложения 3.1}

Из (2.2) и (3.3) следует, что разность $\delta=w-u$ удовлетворяет уравнению

$$
\frac{d \delta}{d s}+\nu A \delta+B(\delta, u)+B(u, \delta)+B(\delta, \delta)=-\mu \nu \kappa_{0}^{2} \mathscr{P} J \delta .
$$

Предположим, что $\delta(\bar{s}) \neq 0$ при некотором $\bar{s} \in \mathbb{R}$. Поскольку $\delta(s)-$ непрерывная функция со значениями в $V$, то найдется некоторый максимальный интервал $\left(s_{0}, s_{1}\right)$, содержащий $\bar{s}$, на котором $\delta(s) \neq 0$ при всех $s \in\left(s_{0}, s_{1}\right)$. Умножая скалярно на $A \delta$ и учитывая соотношения $(2.8),(2.9),(2.18)$, получаем при всех $s \in\left(s_{0}, s_{1}\right)$

$$
\begin{aligned}
& \frac{1}{2} \frac{d}{d s}\|\delta\|^{2}+\nu|A \delta|^{2}=-(B(\delta, u), A \delta)-(B(u, \delta), A \delta)-\mu \nu \kappa_{0}^{2}(\mathscr{P} J \delta, \delta) \\
& =(B(\delta, \delta), A u)-\mu \nu \kappa_{0}^{2}(J \delta-\delta, A \delta)-\mu \nu \kappa_{0}^{2}\|\delta\|^{2} \\
& \leqslant c_{T}\|\delta\|^{2}\left(1+\log \frac{|A \delta|^{2}}{\kappa_{0}^{2}\|\delta\|^{2}}\right)|A u|+\mu \nu \kappa_{0}^{2} c_{1} h^{2}|A \delta|^{2} \\
& \quad+\mu \nu \kappa_{0}^{2} c_{2} h\|\delta\||A \delta|-\mu \nu \kappa_{0}^{2}\|\delta\|^{2} \\
& \leqslant c_{T}\|\delta\|^{2}\left(1+\log \frac{|A \delta|^{2}}{\kappa_{0}^{2}\|\delta\|^{2}}\right)|A u|+\mu \nu \kappa_{0}^{2} c_{J} h^{2}|A \delta|^{2}-\frac{1}{2} \mu \nu \kappa_{0}^{2}\|\delta\|^{2} .
\end{aligned}
$$

Далее, применяя (3.5), имеем

$$
\frac{d}{d s}\|\delta\|^{2}+\nu \kappa_{0}^{2}\left[\mu+\frac{|A \delta|^{2}}{\kappa_{0}^{2}\|\delta\|^{2}}-\frac{2 c_{T}|A u|}{\nu \kappa_{0}^{2}}\left(1+\log \frac{|A \delta|^{2}}{\kappa_{0}^{2}\|\delta\|^{2}}\right)\right]\|\delta\|^{2} \leqslant 0,
$$

т. e.

$$
\frac{d}{d s}\|\delta\|^{2}+\nu \kappa_{0}^{2}[\mu+\theta-\alpha(1+\log \theta)]\|\delta\|^{2} \leqslant 0,
$$

где

$$
\theta=\frac{|A \delta|^{2}}{\kappa_{0}^{2}\|\delta\|^{2}} \geqslant 1, \quad \alpha=\frac{2 c_{T}|A u|}{\nu \kappa_{0}^{2}} .
$$

Найдем нижнюю грань $\psi(\theta)=\theta-\alpha(1+\log \theta)$ по всем $\theta \geqslant 1$. Отметим, что

$$
\psi(1)=1-\alpha, \quad \lim _{\theta \rightarrow \infty} \psi(\theta)=\infty
$$

при этом $\psi$ убывает при $\theta<\alpha$ и возрастает при $\theta>\alpha$. Таким образом,

$$
\min _{\theta \geqslant 1} \psi(\theta)= \begin{cases}\psi(1)=1-\alpha \geqslant 0, & \text { если } 0 \leqslant \alpha \leqslant 1 \\ \psi(\alpha)=-\alpha \log \alpha \geqslant 0, & \text { если } \alpha \geqslant 1 .\end{cases}
$$

Далее, $1-\alpha \geqslant-\alpha \log \alpha$ при $\alpha \in(0,1]$. Действительно, легко убедиться, что для $\eta(\alpha)=1-\alpha+\alpha \log \alpha$ выполнены соотношения $\eta\left(0^{+}\right)=1, \eta(1)=0$ и $\eta^{\prime}(\alpha)=\log \alpha \leqslant 0$. Как следствие,

$$
\min _{\theta \geqslant 1} \psi(\theta) \geqslant-\alpha \log \alpha
$$


Применяя (4.2) и затем (2.22) к (4.1), мы получаем, что

$$
\frac{d}{d s}\|\delta\|^{2}+\nu \kappa_{0}^{2}\left[\mu-\frac{2 c_{T}|A u|}{\nu \kappa_{0}^{2}} \log \left(2 c_{T} c_{0} G^{3}\right)\right]\|\delta\|^{2} \leqslant 0,
$$

поскольку $u \in \mathscr{A}$. Следовательно,

$$
\|\delta(s)\|^{2} \leqslant \exp \left\{\left[-\nu \kappa_{0}^{2} \mu+\frac{6 c_{T} \log \left(c_{3} G\right)}{s-\sigma_{0}} \int_{\sigma_{0}}^{s}|A u| d \tau\right]\left(s-\sigma_{0}\right)\right\}\left\|\delta\left(\sigma_{0}\right)\right\|^{2},
$$

где $c_{3}=\left(2 c_{T} c_{0}\right)^{1 / 3}$ и $s_{0}<\sigma_{0}<s<s_{1}$. Если $s_{0}>-\infty$, то $\delta\left(s_{0}\right)=0$, и, полагая $\sigma_{0} \rightarrow s_{0}^{+}$, мы заключаем, что $\delta(s)=0$ при $s \in\left(s_{0}, s_{1}\right)$. В противном случае из (2.22) следует, что

$$
\|\delta(s)\|^{2} \leqslant \exp \left\{\nu \kappa_{0}^{2}\left[-\mu+6 c_{T} \log \left(c_{3} G\right) G\right]\left(s-\sigma_{0}\right)\right\}\left\|\delta\left(\sigma_{0}\right)\right\|^{2}
$$

при достаточно большом $\left|\sigma_{0}\right|$. При $\sigma_{0} \rightarrow-\infty$ из $(3.4)$ вытекает, что $\delta(s)=0$. Так как $s \in\left(s_{0}, s_{1}\right)$ произвольно, то, в частности, $\delta(\bar{s})=0$. Полученное противоречие доказывает предложение 3.1 .

\section{5. Доказательство теоремы 3.2}

В данном разделе мы даем формальное доказательство для каждой из оценок, сформулированных в теореме 3.2. При этом мы указываем путь к получению строгого обоснования существования решения $w$ уравнения (3.6), для которого, вместе с $w^{\prime}$, выполнены эти оценки. Рассмотрим следующие галёркинские приближения для (3.6):

$$
\frac{d w_{n}}{d s}+\nu A w_{n}+P_{n} B\left(w_{n}, w_{n}\right)=P_{n} f-\mu \nu \kappa_{0}^{2} P_{n} \mathscr{P}\left(J w_{n}-v\right),
$$

где $P_{n}$ - ортогональная проекция пространства $H$ на подпространство $H_{n}:=$ $\operatorname{span}\left\{w_{1}, w_{2}, \ldots, w_{n}\right\}$ (линейную оболочку первых $n$ собственных функций оператора Стокса $A)$. Имеет место следующий результат.

ПредлОЖениЕ 5.1. Уравнение (5.1) имеет решение $w_{n}(s)$ при всех $s \in \mathbb{R}$. Это решение, вместе с $\left(d w_{n} / d s\right)(s)$, удовлетворяет всем оценкам из теоремы 3.2 .

ДоказАтельство. Пусть $k \in \mathbb{N}$. Дополнив систему (5.1) начальными данными $w_{n}\left(-k \nu \kappa_{0}^{2}\right)=0$, мы получим конечную систему обыкновенных дифференциальных уравнений с квадратичной полиномиальной нелинейностью. При таком выборе начальных данных система (5.1) имеет единственное решение $w_{n, k}$ на коротком интервале времени, симметричном относительно начального момента времени $s=-k \nu \kappa_{0}^{2}$. При этом на этом малом интервале $d w_{n, k} / d s$ является единственным решением задачи Коши

$$
\begin{gathered}
\frac{d \widetilde{w}_{n}}{d s}+\nu A \widetilde{w}_{n}+P_{n} B\left(\widetilde{w}_{n}, w_{n}\right)+P_{n} B\left(w_{n}, \widetilde{w}_{n}\right)=-\mu \nu \kappa_{0}^{2} P_{n} \mathscr{P}\left(J \widetilde{w}_{n}-v^{\prime}\right), \\
\widetilde{w}_{n}\left(-k \nu \kappa_{0}^{2}\right)=P_{n} f+\mu \nu \kappa_{0}^{2} P_{n} \mathscr{P} v .
\end{gathered}
$$

Рассматривая бесконечный полуинтервал $\left[-k \nu \kappa_{0}^{2}, \infty\right)$ и используя те же аргументы, которые будут использованы нами ниже при получении оценок для $\|w\|$, 
мы показываем, что такие же оценки верны и для $\left\|w_{n, k}(s)\right\|$ при $s \geqslant-k \nu \kappa_{0}^{2}$. Таким образом, решения $w_{n, k}(s)$ остаются ограниченными при всех $s \geqslant-k \nu \kappa_{0}^{2}$ и, следовательно, являются решениями (5.1) при $s \in\left[-k \nu \kappa_{0}^{2}, \infty\right)$. Далее, поскольку величина $\left|A\left(\left(d w_{n, k} / d s\right)\left(-k \nu \kappa_{0}^{2}\right)\right)\right|=\left|A\left(P_{n} f+\mu \nu \kappa_{0}^{2} P_{n} \mathscr{P} v\right)\right|$ конечна и не зависит от $k$, можно показать, используя те же рассуждения, которые будут использованы нами ниже при получении оценок для $\left\|w^{\prime}\right\|$ и $\left|A w^{\prime}\right|$, что при всех $s \in\left[-k \nu \kappa_{0}^{2}, \infty\right)$ для $\left|A\left(\left(d w_{n, k} / d s\right)(s)\right)\right|$ имеет место равномерная оценка, не зависящая от $k$.

Теперь пусть $j \in \mathbb{N}$. Используя критерий компактности Арцела-Асколи, можно найти подпоследовательность $w_{n, k(j)}$ последовательности $w_{n, k}$, сходящуюся при $k(j) \rightarrow \infty$ к $w_{n}^{j}$, являющемуся решением системы (5.1) на интервале $\left[-j \nu \kappa_{0}^{2}, j \nu \kappa_{0}^{2}\right]$. При этом для $w_{n}^{j}(s)$ и $\left(d w_{n}^{j} / d s\right)(s)$ выполнены все оценки из теоремы 3.2 при всех $s \in\left[-j \nu \kappa_{0}^{2}, j \nu \kappa_{0}^{2}\right]$. Окончательно, используя стандартную канторовскую диагональную процедуру, мы показываем, что $w_{n, k(k)}$ сходится к $w_{n}$ при $k \rightarrow \infty$ и что $w_{n}$ удовлетворяет требуемым свойствам из предложения 5.1. Предложение 5.1 доказано.

Продолжим доказательство теоремы. Учитывая предложение 5.1, мы воспользуемся теоремой Обена о компактности (см., например, [6], [22]), из которой следует, что при любых $m \in \mathbb{N}$ существует подпоследовательность $w_{n(m)}$ последовательности $w_{n}$, сходящаяся к $w^{(m)}$ в соответствующих пространствах на интервале $\left[-\nu \kappa_{0} m, \nu \kappa_{0} m\right]$ при $n(m) \rightarrow \infty$. Переходя к пределу и используя те же аргументы, что при рассмотрении двумерной системы Навье-Стокса, мы показываем, что $w^{(m)}$ является решением уравнения $(3.6)$ в интервале $\left[-\nu \kappa_{0} m\right.$, $\left.\nu \kappa_{0} m\right]$. Вдобавок, $w^{(m)}$ и $\left(d w^{(m)} / d s\right)(s)$ удовлетворяют оценкам из теоремы 3.2 на интервале $\left[-\nu \kappa_{0} m, \nu \kappa_{0} m\right]$. Используя еще раз диагональную процедуру, мы показываем, что диагональная подпоследовательность $w_{n(n)}$ сходится при $n \rightarrow \infty$ к решению $w$ уравнения (3.6). При этом $w$ и $w^{\prime}$ удовлетворяют всем оценкам теоремы 3.2 при всех $s \in \mathbb{R}$. Этим мы заканчиваем формальное обоснование оценок, которые будут получены нами ниже.

5.1. Оценка для $\|w\|$. Умножая скалярно (3.6) на $A w$ и учитывая (2.8), имеем

$$
\frac{1}{2} \frac{d}{d s}\|w\|^{2}+\nu|A w|^{2}=(f, A w)+\mu \nu \kappa_{0}^{2}(v, A w)-\mu \nu \kappa_{0}^{2}(J w-w, A w)-\mu \nu \kappa_{0}^{2}\|w\|^{2} .
$$

Далее, используя (3.1), мы получаем, что

$$
\begin{gathered}
\frac{1}{2} \frac{d}{d s}\|w\|^{2}+\nu|A w|^{2} \leqslant \\
\frac{|f|^{2}}{2 \nu}+\frac{\nu}{2}|A w|^{2}+\mu \nu \kappa_{0}^{2}\|v\|^{2}+\frac{\mu \nu \kappa_{0}^{2}}{4}\|w\|^{2}-\mu \nu \kappa_{0}^{2}\|w\|^{2} \\
+\frac{\mu \nu \kappa_{0}^{2}}{4}\|w\|^{2}+\mu \nu \kappa_{0}^{2} c_{1}^{2} h^{2}|A w|^{2}+\mu \nu \kappa_{0}^{2} c_{2} h^{2}|A w|^{2}
\end{gathered}
$$

откуда

$$
\frac{d}{d s}\|w\|^{2}+\mu \nu \kappa_{0}^{2}\|w\|^{2}+\nu\left(1-2 \mu h^{2} \kappa_{0}^{2}\left(c_{1}^{2}+c_{2}\right)\right)|A w|^{2} \leqslant \frac{|f|^{2}}{\nu}+2 \mu \nu \kappa_{0}^{2}\|v\|^{2} .
$$

Следовательно, если мы предположим, что $h$ достаточно мало, чтобы для него было выполнено неравенство

$$
2 \mu h^{2} \kappa_{0}^{2}\left(c_{1}^{2}+c_{2}\right)<\frac{1}{2},
$$


то, используя неравенство Гронуолла и предположение, что норма $\|w(s)\|$ ограничена, мы приходим к следующей оценке:

$$
\|w(s)\|^{2} \leqslant 2 \nu^{2} \kappa_{0}^{2}\|v\|_{X}^{2}+\frac{|f|^{2}}{\mu \nu^{2} \kappa_{0}^{2}} \leqslant \nu^{2} \kappa_{0}^{2}\left(2 \rho^{2}+\frac{G^{2}}{\mu}\right) \leqslant \nu^{2} \kappa_{0}^{2} K^{2} .
$$

Далее, рассмотрим эволюционное уравнение для $w^{\prime}=d w / d s$ :

$$
\frac{d w^{\prime}}{d s}+\nu A w^{\prime}+B\left(w^{\prime}, w\right)+B\left(w, w^{\prime}\right)=-\mu \nu \kappa_{0}^{2} \mathscr{P}\left(J w^{\prime}-v^{\prime}\right) .
$$

5.2. Оценка для $\left\|w^{\prime}\right\|$. Умножая скалярно (5.6) на $A w^{\prime}$, учитывая (2.9), (3.1) и рассуждая аналогично, имеем, что

$$
\begin{gathered}
\frac{d}{d s}\left\|w^{\prime}\right\|^{2}+\mu \nu \kappa_{0}^{2}\left\|w^{\prime}\right\|^{2}+2 \nu\left(1-\mu h^{2} \kappa_{0}^{2}\left(c_{1}^{2}+c_{2}\right)\right)\left|A w^{\prime}\right|^{2} \\
\leqslant 2\left|\left(B\left(w^{\prime}, w^{\prime}\right), A w\right)\right|+2 \mu \nu \kappa_{0}^{2}\left\|v^{\prime}\right\|^{2} \\
\leqslant 2\left\|w^{\prime}\right\|_{L^{\infty}}\left\|w^{\prime}\right\||A w|+2 \mu \nu \kappa_{0}^{2}\left\|v^{\prime}\right\|^{2} .
\end{gathered}
$$

Применим (2.28) к $\left\|w^{\prime}\right\|_{L^{\infty}}$. Это дает

$$
\begin{aligned}
\left\|w^{\prime}\right\|_{L^{\infty}}\left\|w^{\prime}\right\||A w| & \leqslant \mathscr{L}_{N}\left\|w^{\prime}\right\|^{2}|A w|+\left(\pi^{1 / 2} \kappa_{0} N\right)^{-1}\left\|w^{\prime}\right\|\left|A w^{\prime}\right||A w| \\
& \leqslant \mathscr{L}_{N}\left\|w^{\prime}\right\|^{2}|A w|+\frac{1}{\nu \kappa_{0}^{2} \pi N^{2}}\left\|w^{\prime}\right\|^{2}|A w|^{2}+\frac{\nu}{4}\left|A w^{\prime}\right|^{2} .
\end{aligned}
$$

Следовательно, с учетом (5.4),

$$
\frac{d}{d s}\left\|w^{\prime}\right\|^{2}+\alpha\left\|w^{\prime}\right\|^{2} \leqslant 2 \mu \nu \kappa_{0}^{2}\left\|v^{\prime}\right\|^{2} \leqslant \mu \nu^{5} \kappa_{0}^{8} K^{2},
$$

где

$$
\alpha=\mu \nu \kappa_{0}^{2}-2 \mathscr{L}_{N}|A w|-\frac{2}{\nu \kappa_{0}^{2} \pi N^{2}}|A w|^{2} .
$$

Из (5.3) и (5.4) вытекает, что

$$
\frac{\nu}{2}|A w(s)|^{2} \leqslant-\frac{d}{d s}\|w(s)\|^{2}+\mu \nu^{3} \kappa_{0}^{4} K^{2},
$$

и, следовательно, учитывая (5.5) и интегрируя, имеем

$$
\int_{s-1 /\left(\nu \kappa_{0}^{2}\right)}^{s}|A w(\sigma)|^{2} d \sigma \leqslant 2(1+\mu) \nu \kappa_{0}^{2} K^{2} .
$$

Из неравенства Коши-Буняковского вытекает, что

$$
\int_{s-1 /\left(\nu \kappa_{0}^{2}\right)}^{s}|A w| d \sigma \leqslant(2(1+\mu))^{1 / 2} K
$$

и, значит,

$$
\int_{s-1 /\left(\nu \kappa_{0}^{2}\right)}^{s} \alpha \geqslant \mu-2^{1 / 2} \frac{(8+2 \pi \log N)^{1 / 2}}{\pi}(1+\mu)^{1 / 2} K-\frac{4(1+\mu)}{\pi N^{2}} K^{2} .
$$

Мы хотим показать, что

$$
\int_{s-1 /\left(\nu \kappa_{0}^{2}\right)}^{s} \alpha(\sigma) d \sigma \geqslant 1 \quad \text { при всех } s \in \mathbb{R} .
$$


Если мы выберем $N^{2}=K(1+\mu)^{1 / 2}$, то тогда (5.9) будет следовать из неравенства

$$
\pi(\mu-1) \geqslant 2^{1 / 2}\left[8+\pi \log K+\frac{\pi}{2} \log (1+\mu)\right]^{1 / 2}(1+\mu)^{1 / 2} K+4(1+\mu)^{1 / 2} K,
$$

которое заведомо выполняется, если

$$
\pi^{2}(\mu-1)^{2} \geqslant 4\left[8+\pi \log K+\frac{\pi}{2} \log (1+\mu)\right](1+\mu) K^{2}+32(1+\mu) K^{2},
$$

что эквивалентно тому, что

$$
\frac{(\mu-1)^{2}}{1+\mu} \geqslant \frac{64}{\pi^{2}} K^{2}+\frac{4}{\pi} K^{2} \log K+\frac{2}{\pi} K^{2} \log (1+\mu) .
$$

С учетом неравенства

$$
\frac{(\mu-1)^{2}}{1+\mu} \geqslant \frac{1+\mu}{4} \quad \forall \mu \geqslant 3
$$

нам достаточно, чтобы было выполнено следующее неравенство:

$$
\frac{1+\mu}{4}\left[1-\frac{8}{\pi} K^{2} \frac{\log (1+\mu)}{1+\mu}\right] \geqslant \frac{64}{\pi^{2}} K^{2}+\frac{4}{\pi}(\log K) K^{2} .
$$

Так как $a \geqslant 2 b \log b, b \geqslant 9$, то $a / \log a \geqslant b$, и мы положим $b=16 K^{2} / \pi, a=1+\mu$. Таким образом, если

$$
1+\mu \geqslant \frac{32 K^{2}}{\pi} \log \frac{16 K^{2}}{\pi},
$$

то нам достаточно, чтобы было выполнено неравенство

$$
\frac{1+\mu}{8} \geqslant \frac{64}{\pi^{2}} K^{2}+\frac{4}{\pi} K^{2} \log K
$$

Итак, (5.9) имеет место, если мы возьмем

$$
\mu \geqslant 80 K^{2} \log K
$$

Снова полагая $N^{2}=K(1+\mu)^{1 / 2}$ и рассуждая аналогично, имеем при $0 \leqslant r \leqslant 1$

$$
\begin{aligned}
\int_{s-r /\left(\nu \kappa_{0}^{2}\right)}^{s} \alpha(\tau) d \tau \geqslant r \mu & -\left[\frac{4}{\pi}-r^{1 / 2} \frac{2^{1 / 2}}{\pi}\right. \\
& \left.\times\left(8+\pi \log K+\frac{\pi}{2} \log (1+\mu)\right)^{1 / 2}\right](1+r \mu)^{1 / 2} K .
\end{aligned}
$$

Таким образом, если

$$
\mu \leqslant c^{\prime} K^{2} \log \left(c^{\prime} K\right)
$$

при некотором $c^{\prime}$, то

$$
\sup _{0 \leqslant r \leqslant 1} \exp \left(-\int_{s-r /\left(\nu \kappa_{0}^{2}\right)}^{s} \alpha\right) \leqslant \exp \left(c K^{2} \log K\right)
$$

с некоторой абсолютной постоянной $c$. В конечном итоге мы выбираем константу $c^{\prime}$ так, чтобы (5.12) было согласовано с (5.11) (см. (5.20)). 
Лемма 5.2. Пусть $\beta \geqslant 0$ - константа, и пусть $y(s) \geqslant 0$ - абсолютно непрерывная ограниченная функиия, для которой выполнено неравенство $y^{\prime}+$ $\alpha y \leqslant \beta$ при всех $s \in \mathbb{R}$. Предположим, что $\int_{s-1 /\left(\nu \kappa_{0}^{2}\right)}^{s} \alpha(\sigma) d \sigma \geqslant 1$ при всех $s \in \mathbb{R}$. Тогда

$$
y(s) \leqslant 2 \frac{\beta}{\nu \kappa_{0}^{2}} \sup _{0 \leqslant r \leqslant 1} \exp \left(-\int_{s-r /\left(\nu \kappa_{0}^{2}\right)}^{s} \alpha(\sigma) d \sigma\right) \quad \text { при всех } s \in \mathbb{R} .
$$

ДокАЗАТЕЛЬСтво. Поскольку $\int_{s-1 /\left(\nu \kappa_{0}^{2}\right)}^{s} \alpha(\sigma) d \sigma \geqslant 1$, то $\int_{-\infty}^{0} \alpha=+\infty$. УМножая неравенство $y^{\prime}+\alpha y \leqslant \beta$ на интегрирующий множитель $\exp \left(\int_{0}^{s} \alpha(\sigma) d \sigma\right)$ и интегрируя от $-\infty$ до $s$, получаем

$$
\begin{aligned}
y(s) & \leqslant \beta \int_{-\infty}^{s} \exp \left(-\int_{\sigma}^{s} \alpha\right) d \sigma \\
& =\beta \int_{-\infty}^{s} \exp \left(-\int_{\sigma+\left\lfloor(s-\sigma) \nu \kappa_{0}^{2}\right\rfloor /\left(\nu \kappa_{0}^{2}\right)}^{s} \alpha \prod_{k=1}^{\left\lfloor(s-\sigma) \nu \kappa_{0}^{2}\right\rfloor} \exp \left(-\int_{\sigma+(k-1) /\left(\nu \kappa_{0}^{2}\right)}^{\sigma+k /\left(\nu \kappa_{0}^{2}\right)} \alpha\right) d \sigma\right. \\
& \leqslant \beta \sup _{0 \leqslant r \leqslant 1} \exp \left(-\int_{s-r /\left(\nu \kappa_{0}^{2}\right)}^{s} \alpha\right) \int_{-\infty}^{s} \prod_{k=1}^{\left\lfloor(s-\sigma) \nu \kappa_{0}^{2}\right\rfloor} e^{-1} d \sigma \\
& =\beta \sup _{0 \leqslant r \leqslant 1} \exp \left(-\int_{s-r /\left(\nu \kappa_{0}^{2}\right)}^{s} \alpha\right) \int_{-\infty}^{s} e^{-\left\lfloor(s-\sigma) \nu \kappa_{0}^{2}\right\rfloor} d \sigma \\
& =\beta \sup _{0 \leqslant r \leqslant 1} \exp \left(-\int_{s-r /\left(\nu \kappa_{0}^{2}\right)}^{s} \alpha\right) \frac{1+e^{-1}+e^{-2}+\cdots}{\nu \kappa_{0}^{2}} \\
& =\frac{2 \beta}{\nu \kappa_{0}^{2}} \sup _{0 \leqslant r \leqslant 1} \exp \left(-\int_{s-r /\left(\nu \kappa_{0}^{2}\right)}^{s} \alpha\right) .
\end{aligned}
$$

Теперь из леммы 5.2 и неравенств (5.9), (5.13) вытекает, что

$$
\left\|w^{\prime}(s)\right\| \leqslant c \nu^{2} \kappa_{0}^{3} \exp \left(c K^{2} \log K\right) \quad \text { при всех } s \in \mathbb{R} \text { и некотором } c>0 \text {. }
$$

5.3. Оценки для $|A w|$ и $\left|A^{3 / 2} w\right|$. Из (5.8) мы получаем

$$
\frac{\nu}{2}|A w|^{2} \leqslant 2\left\|w^{\prime}\right\|\|w\|+\mu \nu^{3} \kappa_{0}^{4} K^{2} \leqslant \frac{1}{\nu \kappa_{0}^{2}}\left\|w^{\prime}\right\|^{2}+\nu \kappa_{0}^{2}\|w\|^{2}+\mu \nu^{3} \kappa_{0}^{4} K^{2},
$$

откуда, с учетом (5.14) и (5.5),

$$
\sup _{s \in \mathbb{R}}|A w(s)| \leqslant c \nu \kappa_{0}^{2} \exp \left(c K^{2} \log K\right) .
$$

Далее, применяя (3.6), находим, что

$$
A^{1 / 2} w^{\prime}+\nu A^{3 / 2} w+A^{1 / 2} B(w, w)=A^{1 / 2} f-\mu \nu \kappa_{0}^{2} A^{1 / 2} \mathscr{P} J w+\mu \nu \kappa_{0}^{2} A^{1 / 2} \mathscr{P} v,
$$

откуда, с учетом (2.24),

$$
\begin{gathered}
\nu\left|A^{3 / 2} w\right| \leqslant\left\|w^{\prime}\right\|+\|f\|+\mu \nu \kappa_{0}^{2}\|v\|+\mu \nu \kappa_{0}^{2}\|\mathscr{P} J w\| \\
+c^{\prime}\left(\left|A^{3 / 4} w\right|^{2}+|w|^{1 / 2}|A w|^{3 / 2}\right) .
\end{gathered}
$$


Так как $h \kappa_{0} \leqslant c$, то из $(3.2)$ имеем

$$
\|\mathscr{P} J w\| \leqslant c(\|w\|+h|A w|) \leqslant c \nu \kappa_{0} \exp \left(c K^{2} \log K\right) .
$$

Применяя (5.12) и (5.15), мы получаем оценку

$$
\sup _{s \in \mathbb{R}}\left|A^{3 / 2} w(s)\right| \leqslant \frac{1}{\nu}\|f\|+\nu \kappa_{0}^{3} c \exp \left(c K^{2} \log K\right) .
$$

5.4. Оценка для $\left|A w^{\prime}\right|$. Умножая уравнение

$$
\frac{d w^{\prime}}{d s}+\nu A w^{\prime}+B\left(w^{\prime}, w\right)+B\left(w, w^{\prime}\right)=-\mu \nu \kappa_{0}^{2} \mathscr{P}\left(J w^{\prime}-v^{\prime}\right)
$$

скалярно на $A^{2} w^{\prime}$, мы приходим к оценке

$$
\begin{aligned}
\frac{1}{2} \frac{d}{d s}\left|A w^{\prime}\right|^{2}+\nu\left|A^{3 / 2} w^{\prime}\right|^{2} \leqslant \mid & \left.\left(B\left(w^{\prime}, w\right), A^{2} w^{\prime}\right)|+|\left(B\left(w, w^{\prime}\right), A^{2} w^{\prime}\right)\left|-\mu \nu \kappa_{0}^{2}\right| A w^{\prime}\right|^{2} \\
& +\mu \nu \kappa_{0}^{2}\left\|J w^{\prime}-w^{\prime}\right\|\left|A^{3 / 2} w^{\prime}\right|+\mu \nu \kappa_{0}^{2}\left\|v^{\prime}\right\|\left|A^{3 / 2} w^{\prime}\right|
\end{aligned}
$$

Оценивая первый нелинейный член, имеем с учетом (2.15), (2.25),

$$
\begin{aligned}
\left|\left(B\left(w^{\prime}, w\right), A^{2} w^{\prime}\right)\right| & =\left|A^{1 / 2} B\left(w^{\prime}, w\right)\right|\left|A^{3 / 2} w^{\prime}\right| \\
\leqslant & \frac{1}{\nu}\left|A^{1 / 2} B\left(w^{\prime}, w\right)\right|^{2}+\frac{\nu}{4}\left|A^{3 / 2} w^{\prime}\right|^{2} \\
\leqslant & \frac{c}{\nu}\left(\left|A^{1 / 2} w^{\prime}\right|\left|A^{1 / 2} w\right|^{1 / 2}\left|A^{3 / 2} w\right|^{1 / 2}\right. \\
& \left.\quad+\left|A^{1 / 4} w^{\prime}\right|\left|A^{5 / 4} w\right|\right)^{2}+\frac{\nu}{4}\left|A^{3 / 2} w^{\prime}\right|^{2} .
\end{aligned}
$$

Для оценки второго нелинейного члена мы проинтегрируем по частям и воспользуемся тем, что $A=-\Delta$ при периодических граничных условиях, откуда, учитывая (2.7), (2.8), (2.13), (2.11), (2.15), имеем

$$
\begin{aligned}
\mid\left(B\left(w, w^{\prime}\right),\right. & \left.A^{2} w^{\prime}\right)|=| \int_{\Omega}\left((w \cdot \nabla) w^{\prime}\right) \cdot \Delta^{2} w^{\prime} d x \mid \\
& \leqslant\left|\int_{\Omega}\left((\Delta w \cdot \nabla) w^{\prime}\right) \cdot \Delta w^{\prime} d x\right|+2\left|\sum_{j=1}^{2} \int_{\Omega}\left(\left(\partial_{j} w \cdot \nabla\right) \partial_{j} w^{\prime}\right) \cdot \Delta w^{\prime} d x\right| \\
& \leqslant c\|\Delta w\|_{L^{4}}\left|\nabla w^{\prime}\right|\left\|\Delta w^{\prime}\right\|_{L^{4}}+c \sum_{j, k=1}^{2}|\nabla w|\left\|\partial_{j} \partial_{k} w^{\prime}\right\|_{L^{4}}^{2} \\
& \leqslant c\left\|\nabla^{2} w\right\|_{H^{1 / 2}}\left\|w^{\prime}\right\|\left\|\Delta w^{\prime}\right\|_{H^{1 / 2}}+c \sum_{j, k=1}^{2}|\nabla w|\left\|\partial_{j} \partial_{k} w^{\prime}\right\|_{H^{1 / 2}}^{2} \\
& \leqslant c|A w|^{1 / 2}\left|A^{3 / 2} w\right|^{1 / 2}\left\|w^{\prime}\right\|\left|A w^{\prime}\right|^{1 / 2}\left|A^{3 / 2} w^{\prime}\right|^{1 / 2}+c\|w\|\left|A w^{\prime}\right|\left|A^{3 / 2} w^{\prime}\right| \\
& \leqslant \frac{c}{\nu}|A w|\left|A^{3 / 2} w\right|\left\|w^{\prime}\right\|^{2}\left|A w^{\prime}\right|+\frac{c}{\nu}\|w\|^{2}\left|A w^{\prime}\right|^{2}+\frac{\nu}{4}\left|A^{3 / 2} w^{\prime}\right|^{2} \\
& \leqslant \frac{c}{\nu^{2} \kappa_{0}^{2}}|A w|^{2}\left|A^{3 / 2} w\right|^{2}\left\|w^{\prime}\right\|^{4} \\
& +\nu \kappa_{0}^{2}\left(1+c K^{2}\right)\left|A w^{\prime}\right|^{2}+\frac{\nu}{4}\left|A^{3 / 2} w^{\prime}\right|^{2} .
\end{aligned}
$$


Окончательно, используя (2.25), (3.2), оценки для $\left\|w^{\prime}\right\|,\left|A^{3 / 2} w\right|$ и неравенство Гронуолла, мы приходим к следующей равномерной оценке:

$$
\left|A w^{\prime}(s)\right| \leqslant \kappa_{0} c \exp \left(c K^{2} \log K\right)\left(\|f\|+\nu^{2} \kappa_{0}^{3}\right) \quad \text { при всех } s \in \mathbb{R}
$$

при условии, что $\mu \geqslant 2\left(1+K^{2}\right)$ (что, в свою очередь, обеспечивается (5.11)).

5.5. Липшицевость $w$ и $w^{\prime}$ в норме $D(A)$. В данном пункте мы докажем единственность ограниченных решений уравнения (3.6) и их непрерывную зависимость от исходной траектории $v \in X$ в указанном ниже смысле. В частности, эти свойства нам потребуются для введения корректно определенного отображения $v \mapsto W(v)$ из пространства $X$ в пространство траекторий, которое определяется формулой $W(v)(s)=w(s), s \in \mathbb{R}$.

Чтобы установить эти свойства, рассмотрим разность $\delta(s)=w_{1}(s)-w_{2}(s)$, где $w_{1}(s), w_{2}(s)$ - две траектории, и запишем оценки для $\delta$ и $\delta^{\prime}$, аналогичные тем, которые мы получили для $w$ и $w^{\prime}$. Единственная сложность возникает только при рассмотрении нелинейного члена. Пусть $\gamma=v_{1}-v_{2}, \widetilde{w}=w_{1}+w_{2}$. Тогда

$$
\frac{d}{d s} \delta+\nu A \delta+\frac{1}{2} B(\widetilde{w}, \delta)+\frac{1}{2} B(\delta, \widetilde{w})=-\mu \nu \kappa_{0}^{2} \mathscr{P}(J \delta-\gamma)
$$

5.6. Оценка для $\|\delta\|$. Умножая скалярно (5.17) на $A \delta$, мы получаем, как в п. 5.1 ,

$$
\begin{gathered}
\frac{1}{2} \frac{d}{d s}\|\delta\|^{2}+\nu|A \delta|^{2}+\mu \nu \kappa_{0}^{2}\|\delta\|^{2}=\mu \nu \kappa_{0}^{2}(\gamma, A \delta)-\mu \nu \kappa_{0}^{2}(J \delta-\delta, A \delta) \\
-\frac{1}{2}(B(\widetilde{w}, \delta), A \delta)-\frac{1}{2}(B(\delta, \widetilde{w}), A \delta) \\
\leqslant \mu \nu \kappa_{0}^{2}\|\gamma\|^{2}+\frac{\mu \nu \kappa_{0}^{2}}{4}\|\delta\|^{2}+\left(c_{T}+c_{B}\right)\|\delta\|\|\widetilde{w}\|\left(\log \frac{e|A \delta|}{\kappa_{0}\|\delta\|}\right)^{1 / 2}|A \delta| \\
+\frac{\mu \nu \kappa_{0}^{2}}{4}\|\delta\|^{2}+\mu \nu \kappa_{0}^{2} c_{1}^{2} h^{2}|A \delta|^{2}+\mu \nu \kappa_{0}^{2} c_{2} h^{2}|A \delta|^{2}
\end{gathered}
$$

где для оценки нелинейных членов мы воспользовались (2.18) и (2.19). Применяя неравенство Юнга к вкладу от нелинейных членов, имеем, с учетом (5.5),

$$
\frac{d}{d s}\|\delta\|^{2}+\nu \kappa_{0}^{2}\left[\mu+\frac{|A \delta|^{2}}{\kappa_{0}^{2}\|\delta\|^{2}}-8\left(c_{T}+c_{B}\right)^{2} K^{2}\left(1+\log \frac{|A \delta|^{2}}{\kappa_{0}^{2}\|\delta\|^{2}}\right)\right]\|\delta\|^{2} \leqslant 2 \mu \nu \kappa_{0}^{2}\|\gamma\|^{2},
$$

если выполнено неравенство (3.5).

На данном шаге мы можем воспользоваться неравенством (4.2) с $\theta=|A \delta|^{2} \times$ $\left(\kappa_{0}^{2}\|\delta\|^{2}\right)^{-1}$ и $\alpha=8\left(c_{T}+c_{B}\right)^{2} K^{2}$, что дает

$$
\frac{d}{d s}\|\delta\|^{2}+\nu \kappa_{0}^{2}\left[\mu-8\left(c_{T}+c_{B}\right)^{2} K^{2} \log \left(8\left(c_{T}+c_{B}\right)^{2} K^{2}\right)\right]\|\delta\|^{2} \leqslant 2 \mu \nu \kappa_{0}^{2}\|\gamma\|^{2} .
$$


Таким образом, если

$$
\mu>32\left(c_{T}+c_{B}\right)^{2} K^{2} \log \left(\sqrt{8}\left(c_{T}+c_{B}\right) K\right),
$$

Tо

$$
\sup _{s \in \mathbb{R}}\|\delta(s)\| \leqslant 4 \nu \kappa_{0}\|\gamma\|_{X}
$$

Для обеспечения совместности (5.11) и (5.18) мы возьмем, как в (3.8),

$$
c_{4} K^{2} \log \left(c_{5} K^{2}\right)<\mu<2 c_{4} K^{2} \log \left(c_{5} K^{2}\right),
$$

где $c_{4}=80\left(c_{T}+c_{B}+1\right)^{2}$ и $c_{5}=\sqrt{8}\left(c_{T}+c_{B}+1\right)$.

5.7. Оценка для $\left\|\delta^{\prime}\right\|$. Запишем уравнение для $\delta^{\prime}$ :

$$
\begin{aligned}
\frac{d}{d s} \delta^{\prime} & +\nu A \delta^{\prime}+\frac{1}{2} B\left(\widetilde{w}^{\prime}, \delta\right)+\frac{1}{2} B\left(\widetilde{w}, \delta^{\prime}\right)+\frac{1}{2} B\left(\delta^{\prime}, \widetilde{w}\right)+\frac{1}{2} B\left(\delta, \widetilde{w}^{\prime}\right) \\
& =-\mu \nu \kappa_{0}^{2}\left(J \delta^{\prime}-\gamma^{\prime}\right) .
\end{aligned}
$$

Умножая скалярно на $A \delta^{\prime}$, мы имеем

$$
\begin{gathered}
\frac{1}{2} \frac{d}{d s}\left\|\delta^{\prime}\right\|^{2}+\nu\left|A \delta^{\prime}\right|^{2}-\frac{1}{2}\left(B\left(\delta^{\prime}, \delta^{\prime}\right), A \widetilde{w}\right)+\frac{1}{2}\left(B\left(\widetilde{w}^{\prime}, \delta\right), A \delta^{\prime}\right)+\frac{1}{2}\left(B\left(\delta, \widetilde{w}^{\prime}\right), A \delta^{\prime}\right) \\
=-\mu \nu \kappa_{0}^{2}\left\|\delta^{\prime}\right\|^{2}+\mu \nu \kappa_{0}^{2}\left(\gamma^{\prime}, A \delta^{\prime}\right)-\mu \nu \kappa_{0}^{2}\left(J \delta^{\prime}-\delta^{\prime}, A \delta^{\prime}\right)
\end{gathered}
$$

В отличие от (5.7) (где мы заменили $w^{\prime}$ на $\delta^{\prime}$ и $w$ на $\widetilde{w}$ ) здесь добавляются два члена: $\left(B\left(\widetilde{w}^{\prime}, \delta\right), A \delta^{\prime}\right)$ и $\left(B\left(\delta, \widetilde{w}^{\prime}\right), A \delta^{\prime}\right)$. Если мы покажем, что они ограничены величиной $\|\gamma\|_{X}$, умноженной на постоянную, то это нам даст липшицевость $w^{\prime}$ при помощи тех же аргументов, что и в п. 5.2.

Для начала отметим, что

$$
\begin{aligned}
& \frac{d}{d s}\left\|\delta^{\prime}\right\|^{2}+\mu \nu \kappa_{0}^{2}\left\|\delta^{\prime}\right\|^{2}+2 \nu\left[1-\mu h^{2} \kappa_{0}^{2}\left(c_{1}^{2}+c_{2}\right)\right]\left|A \delta^{\prime}\right|^{2} \\
& \quad \leqslant\left|\left(B\left(\delta^{\prime}, \delta^{\prime}\right), A \widetilde{w}\right)\right|+\left|\left(B\left(\widetilde{w}^{\prime}, \delta\right), A \delta^{\prime}\right)\right|+\left|\left(B\left(\delta, \widetilde{w}^{\prime}\right), A \delta^{\prime}\right)\right|+2 \mu \nu \kappa_{0}^{2}\left\|\gamma^{\prime}\right\|^{2} .
\end{aligned}
$$

Следовательно,

$$
\begin{aligned}
\left|\left(B\left(\delta^{\prime}, \delta^{\prime}\right), A \widetilde{w}\right)\right| & \leqslant\left\|\delta^{\prime}\right\|_{L^{\infty}}\left\|\delta^{\prime}\right\||A \widetilde{w}| \\
& \leqslant \mathscr{L}_{N}\left\|\delta^{\prime}\right\||A \widetilde{w}|+\left(\pi^{1 / 2} \kappa_{0} N\right)^{-1}\left\|\delta^{\prime}\right\|\left|A \delta^{\prime}\right||A \widetilde{w}| \\
& \leqslant \mathscr{L}_{N}\left\|\delta^{\prime}\right\||A \widetilde{w}|+\frac{\left\|\delta^{\prime}\right\|^{2}|A \widetilde{w}|^{2}}{2 \pi \nu \kappa_{0}^{2} N^{2}}+\frac{\nu}{2}\left|A \delta^{\prime}\right|^{2}
\end{aligned}
$$

и (по (2.16))

$$
\begin{aligned}
\left|\left(B\left(\delta, \widetilde{w}^{\prime}\right), A \delta^{\prime}\right)\right| & \leqslant c_{\mathrm{L}}|\delta|^{1 / 2}\|\delta\|^{1 / 2}\left\|\widetilde{w}^{\prime}\right\|^{1 / 2}\left|A \widetilde{w}^{\prime}\right|^{1 / 2}\left|A \delta^{\prime}\right| \\
& \leqslant \frac{\nu}{4}\left|A \delta^{\prime}\right|^{2}+c_{\mathrm{L}}^{2} \nu^{-1} \kappa_{0}^{-2}\|\delta\|^{2}\left|A \widetilde{w}^{\prime}\right|^{2} .
\end{aligned}
$$


При этом, применяя (2.17), мы получаем

$$
\begin{aligned}
\left|\left(B\left(\widetilde{w}^{\prime}, \delta\right), A \delta^{\prime}\right)\right| & \leqslant c_{\mathrm{A}}\left|\widetilde{w}^{\prime}\right|^{1 / 2}\left|A \widetilde{w}^{\prime}\right|^{1 / 2}\|\delta\|\left|A \delta^{\prime}\right| \\
& \leqslant \frac{\nu}{4}\left|A \delta^{\prime}\right|^{2}+c_{\mathrm{A}}^{2} \nu^{-1} \kappa_{0}^{-2}\|\delta\|^{2}\left|A \widetilde{w}^{\prime}\right|^{2} .
\end{aligned}
$$

Таким образом,

$$
\begin{aligned}
& \frac{d}{d s}\left\|\delta^{\prime}\right\|^{2}+\mu \nu \kappa_{0}^{2}\left\|\delta^{\prime}\right\|^{2}+\nu\left[1-2 \mu h^{2} \kappa_{0}^{2}\left(c_{1}^{2}+c_{2}\right)\right]\left|A \delta^{\prime}\right|^{2} \\
& \quad \leqslant \mathscr{L}_{N}\left\|\delta^{\prime}\right\||A \widetilde{w}|+\frac{\left\|\delta^{\prime}\right\|^{2}|A \widetilde{w}|^{2}}{2 \pi \nu \kappa_{0}^{2} N^{2}}+2 \mu \nu \kappa_{0}^{2}\left\|\gamma^{\prime}\right\|^{2}+\left(c_{\mathrm{A}}^{2}+c_{\mathrm{L}}^{2}\right) \nu^{-1} \kappa_{0}^{-2}\|\delta\|^{2}\left|A \widetilde{w}^{\prime}\right|^{2} \\
& \quad \leqslant \mathscr{L}_{N}\left\|\delta^{\prime}\right\||A \widetilde{w}|+\frac{\left\|\delta^{\prime}\right\|^{2}|A \widetilde{w}|^{2}}{2 \pi \nu \kappa_{0}^{2} N^{2}}+2 \mu \nu^{5} \kappa_{0}^{8}\|\gamma\|_{X}^{2}+\left(c_{\mathrm{A}}^{2}+c_{\mathrm{L}}^{2}\right) \nu^{-1} \kappa_{0}^{-2}\|\delta\|^{2}\left|A \widetilde{w}^{\prime}\right|^{2} .
\end{aligned}
$$

С учетом (3.5) мы можем избавиться от члена, содержащего $\left|A \delta^{\prime}\right|^{2}$. Применяя (5.16) к неравенству $\left|A \widetilde{w}^{\prime}\right| \leqslant\left|A w_{1}^{\prime}\right|+\left|A w_{2}^{\prime}\right|$ и учитывая (5.19), имеем

$$
\frac{d}{d s}\left\|\delta^{\prime}\right\|^{2}+\alpha\left\|\delta^{\prime}\right\|^{2} \leqslant \beta
$$

где

$$
\alpha=\mu \nu \kappa_{0}^{2}-\mathscr{L}_{N}\left\|\delta^{\prime}\right\||A \widetilde{w}|+\frac{|A \widetilde{w}|^{2}}{2 \pi \nu \kappa_{0}^{2} N^{2}}
$$

и

$$
\beta=2 \mu \nu^{5} \kappa_{0}^{8}\|\gamma\|_{X}^{2}+32 c \nu \kappa_{0}^{2}\left(c_{\mathrm{A}}^{2}+c_{\mathrm{L}}^{2}\right) \exp \left(c K^{2} \log K\right)\left(\|f\|+\nu^{2} \kappa_{0}^{3}\right)^{2}\|\gamma\|_{X}^{2} .
$$

Действуя, как в п. 5.2, имеем

$$
\sup _{s \in \mathbb{R}}\left\|\delta^{\prime}(s)\right\| \leqslant\left(\|f\|+\nu^{2} \kappa_{0}^{3}\right) C\left\|_{\gamma}\right\|_{X},
$$

где $C=c \exp \left(c K^{2} \log K\right)$ с некоторой универсальной постоянной $c$. Отметим еще раз, что (5.9) обеспечивается (5.12), так что нет необходимости менять границы изменения $\mu$ в неравенстве (5.12).

5.8. Оценка для $|A \delta|$. Из (5.17), с учетом (2.16), (2.17), (3.1), имеем

$$
\begin{aligned}
\nu|A \delta| \leqslant\left|\delta^{\prime}\right| & +\frac{c_{\mathrm{A}}+c_{\mathrm{L}}}{2 \kappa_{0}}|A \widetilde{w}|\|\delta\|+\mu \nu \kappa_{0}^{2}|\gamma| \\
& +\mu \nu \kappa_{0}^{2}|\delta|+\mu h \nu c_{1} \kappa_{0}^{2}\|\delta\|+\mu h^{2} c_{2} \nu \kappa_{0}^{2}|A \delta|
\end{aligned}
$$

откуда

$$
\nu\left(1-\mu h^{2} c_{2} \kappa_{0}^{2}\right)|A \delta| \leqslant \kappa_{0}^{-1}\left\|\delta^{\prime}\right\|+\mu \nu \kappa_{0}\|\gamma\|\left[\mu \nu \kappa_{0}\left(1+h c_{1} \kappa_{0}\right)+\frac{c_{\mathrm{A}}+c_{\mathrm{L}}}{2 \kappa_{0}}|A \widetilde{w}|\right]\|\delta\| .
$$

Таким образом, если выполнено (3.5), то из (5.15), (5.19) следует, что

$$
\sup _{s \in \mathbb{R}}|A \delta(s)| \leqslant \frac{C}{\nu \kappa_{0}}\left(\|f\|+\nu^{2} \kappa_{0}^{3}\right)\|\gamma\|_{X},
$$

где $C=c \exp \left(c K^{2} \log K\right)$ с некоторой универсальной постоянной $c>0$. 


\section{Список литературы}

[1] A. Azouani, E. Olson, E.S. Titi, Continuous data assimilation using general interpolant observables, 2013, 25 pp., arXiv: 1304.0997; J. Nonlinear Sci. (to appear).

[2] A. Azouani, E.S. Titi, Feedback control of nonlinear dissipative systems by finite determining parameters - a reaction-diffusion paradigm, 2013, 21 pp., arXiv: 1301.6992.

[3] Дж. К. Бэтчелор, Теория однородной турбулентности, ИЛ, М., 1955, 200 с.; пер. с англ.: G. K. Batchelor, The theory of homogeneous turbulence, Cambridge Monogr. Mech. Appl. Math., Cambridge Univ. Press, Cambridge, 1953, x+197 pp.

[4] H. Brézis, T. Gallouet, "Nonlinear Schrödinger evolution equations", Nonlinear Anal., 4:4 (1980), 677-681.

[5] B. Cockburn, D. Jones, E. S. Titi, "Estimating the number of asymptotic degrees of freedom for nonlinear dissipative systems", Math. Comp., 66:219 (1997), 1073-1087.

[6] P. Constantin, C. Foias, Navier-Stokes equations, Chicago Lectures in Math., Univ. Chicago Press, Chicago, IL, 1988, x+190 pp.

[7] P. Constantin, C. Foias, R. Temam, "On the dimension of the attractors in two-dimensional turbulence", Phys. D, 30:3 (1988), 284-296.

[8] C. Foias, M.S. Jolly, R. Kravchenko, E.S. Titi, "A determining form for the 2D Navier-Stokes equations: The Fourier modes case", J. Math. Phys., 53:11 (2012), 115623, $30 \mathrm{pp}$.

[9] C. Foias, M. Jolly, R. Lan, R. Rupam, Y. Yang, Time analyticity with higher norm estimates for the 2D Navier-Stokes equations, 2013, arXiv: 1312.0929; IMA J. Appl. Math. (to appear).

[10] C. Foias, O. Manley, R. Rosa, R. Temam, "Navier-Stokes equations and turbulence", Encyclopedia Math. Appl., 83, Cambridge Univ. Press, Cambridge, 2001, xiv+347 pp.

[11] C. Foiaş, G. Prodi, "Sur le comportement global des solutions non-stationnaires des équations de Navier-Stokes en dimension 2", Rend. Sem. Mat. Univ. Padova, 39 (1967), 1-34.

[12] C. Foias, G. R. Sell, R. Temam, "Inertial manifolds for nonlinear evolutionary equations", J. Differential Equations, 73:2 (1988), 309-353.

[13] C. Foias, G. R. Sell, E. S. Titi, "Exponential tracking and approximation of inertial manifolds for dissipative nonlinear equations", J. Dynam. Differential Equations, 1:2 (1989), 199-244.

[14] C. Foias, R. Temam, "Asymptotic numerical analysis for the Navier-Stokes equations", Nonlinear dynamics and turbulence, Interaction Mech. Math. Ser., Pitman, Boston, MA, 1983, 139-155.

[15] C. Foias, R. Temam, "Determination of the solutions of the Navier-Stokes equations by a set of nodal values", Math. Comp., 43:167 (1984), 117-133.

[16] J. K. Hale, Asymptotic behavior of dissipative systems, Math. Surveys Monogr., 25, Amer. Math. Soc., Providence, RI, 1988, x+198 pp.

[17] D. A. Jones, E. S. Titi, "Determining finite volume elements for the 2D Navier-Stokes equations", Phys. D, 60:1-4 (1992), 165-174.

[18] D. A. Jones, E.S. Titi, "Upper bounds on the number of determining modes, nodes and volume elements for the Navier-Stokes equations", Indiana Univ. Math. J., 42:3 (1993), 875-887.

[19] R. H. Kraichnan, "Inertial ranges in two-dimensional turbulence", Phys. Fluids, 10:7 (1967), 1417-1423.

[20] C. E. Leith, "Diffusion approximation for two-dimensional turbulence", Phys. Fluids, 11:3 (1968), 671-673. 
[21] V.X. Liu, "A sharp lower bound for the Hausdorff dimension of the global attractors of the 2D Navier-Stokes equations", Comm. Math. Phys., 158:2 (1993), 327-339.

[22] R. Temam, Infinite-dimensional dynamical systems in mechanics and physics, 2nd ed., Appl. Math. Sci., 68, Springer-Verlag, New York, 1997, xxii+648 pp.

[23] E. S. Titi, "On a criterion for locating stable stationary solutions to the Navier-Stokes equations", Nonlinear Anal., 11:9 (1987), 1085-1102.

\section{Киприан Фояш}

(Ciprian Foias)

Texas A\&M University, College Station, USA

E-mail: foias@math.tamu.edu

\section{Майкл С. Джолли}

(Michael S. Jolly)

Indiana University, Bloomington, USA

E-mail: msjolly@indiana.edu

\section{Ростислав Кравченко}

(Rostyslav Kravchenko)

Chicago University, Chicago, USA

E-mail: rkchenko@gmail.com

\section{Эдрисс С. Тити}

(Edriss S. Titi)

Weizmann Institute of Science, Rehovot, Israel;

University of California, Irvine, USA

E-mail: etiti@math.uci.edu
Поступила в редакцию

27.10 .2013 